\title{
ON THE FINITE ENERGY WEAK SOLUTIONS TO A SYSTEM IN QUANTUM FLUID DYNAMICS
}

\author{
PAOLO ANTONELLI AND PIERANGELO MARCATI
}

\begin{abstract}
In this paper we consider the global existence of weak solutions to a class of Quantum Hydrodynamics (QHD) systems with initial data, arbitrarily large in the energy norm. These type of models, initially proposed by Madelung [40], have been extensively used in Physics to investigate Supefluidity and Superconductivity phenomena [19], [35] and more recently in the modeling of semiconductor devices [20] . Our approach is based on various tools, namely the wave functions polar decomposition, the construction of approximate solution via a fractional steps method which iterates a Schrödinger Madelung picture with a suitable wave function updating mechanism. Therefore several a priori bounds of energy, dispersive and local smoothing type allow us to prove the compactness of the approximating sequences. No uniqueness result is provided.
\end{abstract}

\section{INTRODUCTION}

In this paper we study the Cauchy problem for the Quantum Hydrodynamics (QHD) system:

(1)

$$
\left\{\begin{array}{l}
\partial_{t} \rho+\operatorname{div} J=0 \\
\partial_{t} J+\operatorname{div}\left(\frac{J \otimes J}{\rho}\right)+\nabla P(\rho)+\rho \nabla V+f(\sqrt{\rho}, J, \nabla \sqrt{\rho})=\frac{\hbar^{2}}{2} \rho \nabla\left(\frac{\Delta \sqrt{\rho}}{\sqrt{\rho}}\right) \\
-\Delta V=\rho-C(x),
\end{array}\right.
$$

with initial data

$$
\rho(0)=\rho_{0}, \quad J(0)=J_{0} .
$$

We are interested to study the global existence in the class of finite energy initial data without higher regularity hypotheses or smallness assumptions.

The analysis of uniqueness of weak solutions in some restricted classes will be done in a forthcoming paper.

We will discuss in particular the case $f(\sqrt{\rho}, J, \nabla \sqrt{\rho})=J$, however we

Date: October 26, 2018. 
are able to treat a more general collision term, as it will be clarified in the following Remark 5. Indeed, we show that it is possible to consider a general collision term of the form $f=\alpha J+\rho \nabla g$, where $\alpha \geq 0$ and $g$ is a nonlinear operator of $\sqrt{\rho}, J, \nabla \sqrt{\rho}$, satisfying certain Carathéodorytype conditions (see Remark 5 for more precise explanations).

There is a formal analogy between (1) and the classical fluid mechanics system, in particular when $\hbar=0$, the system (1) formally coincides with the (nonhomogeneous) Euler-Poisson incompressible fluid system. The theoretical description of microphysical systems is generally based on the wave mechanics of Schrödinger , the matrix mechanics of Heisenberg or the path-integral mechanics of Feynman (see [19]). Another approach to Quantum mechanics was taken by Madelung and de Broglie (see [40]), in particular the hydrodynamic theory of quantum mechanics has been later extended by de Broglie (the idea of "double solution") and used as a scheme for quasicausal interpretation of microphysical systems. There is an extensive literature (see for example [12], [35], [32], [31], [34], [30], and references therein) where superfluidity phenomena are described by means of quantum hydrodynamic systems. Furthermore, the Quantum Hydrodynamics system is well known in literature since it has been used in modeling semiconductor devices at nanometric scales (see [20]). The hydrodynamical formulation for quantum mechanics is quite useful with respect to other descriptions for semiconductor devices, such as those based on Wigner-Poisson or Schrödinger-Poisson, since kinetic or Schrödinger equations are computationally very expensive. For a derivation of the QHD system we refer to [2], [15], [28], [29], [21], [13], [14].

The unknowns $\rho, J$ represent the charge and the current densities respectively, $P(\rho)$ the classical pressure which we assume to satisfy $P(\rho)=$ $\frac{p-1}{p+1} \rho^{\frac{p+1}{2}}$ (here and throughout the paper we assume $1 \leq p \leq 5$ ). The function $V$ is the self-consistent electric potential, given by the Poisson equation, the function $C(x)$ represents the density of the background positively charged ions. In the paper we will treat extensively the case $C(x)=0$, but all the results can be extended to more general cases. For instance we can assume $C \in W^{1,1}\left(\mathbb{R}^{3}\right) \cap W^{1,3}\left(\mathbb{R}^{3}\right)$.

The term $\frac{\hbar^{2}}{2} \rho \nabla\left(\frac{\Delta \sqrt{\rho}}{\sqrt{\rho}}\right)$ can be interpreted as the quantum Bohm potential, or as a quantum correction to the pressure, indeed with some regularity assumptions we can write the dispersive term in different 
ways:

$$
\begin{aligned}
\frac{\hbar^{2}}{2} \rho \nabla\left(\frac{\Delta \sqrt{\rho}}{\sqrt{\rho}}\right)=\frac{\hbar^{2}}{4} \operatorname{div}\left(\rho \nabla^{2}\right. & \log \rho) \\
& =\frac{\hbar^{2}}{4} \Delta \nabla \rho-\hbar^{2} \operatorname{div}(\nabla \sqrt{\rho} \otimes \nabla \sqrt{\rho}) .
\end{aligned}
$$

There is a formal equivalence between the system (1) and the following nonlinear Schrödinger-Poisson system:

$$
\left\{\begin{array}{l}
i \hbar \partial_{t} \psi+\frac{\hbar^{2}}{2} \Delta \psi=|\psi|^{p-1} \psi+V \psi+\tilde{V} \psi \\
-\Delta V=|\psi|^{2}
\end{array}\right.
$$

where $\tilde{V}=\frac{1}{2 i} \log \left(\frac{\psi}{\psi}\right)$, in particular the hydrodynamic system (1) can be obtained by defining $\rho=|\psi|^{2}, J=\hbar \operatorname{Im}(\bar{\psi} \nabla \psi)$ and by computing the related balance laws.

This problem has to face a serious mathematical difficulty connected with the need to solve (4) with the ill-posed potential $\tilde{V}$. Presently there are no mathematical results concerning the solutions to (4), except small perturbations around constant plane waves or local existence results under various severe restrictions (see [27], [36]). In the paper by $\mathrm{Li}$ and the second author [37] there is a global existence result for the system (1), regarding small perturbations in higher Sobolev norms of subsonic stationary solutions, with periodic boundary conditions.

A possible way to circumvent this type of difficulty could be to develop a theory regarding wave functions taking values on Riemann manifolds but we will not pursue this direction in this paper (see also [10], [46]). Another nontrivial problem concerning the derivation of solutions to (1) starting from the solutions to (4), regards the reconstruction of the initial datum $\psi(0)$ in terms of the observables $\rho(0), J(0)$. Actually this is a case of a more general important problem in physics, pointed out by Weigert in [53]. He named it the Pauli problem (since this question originated from a footnote in Pauli's article in Handbuch der Physik, see [43]), and it regards the possibility of reconstructing a pure quantum state, just by knowing a finite set of measurements of the state (in our case, the mass and current densities). Here the possible existence of nodal regions, or vacuum in fluid terms, namely where $\rho=0$, forbids in general this reconstruction in a classical way, and in any case some additional requirements (quantization rules like the Bohr-Sommerfeld rule) would be necessary. In any case, various authors (see [53] and references therein) showed that knowledge of only position and momentum distribution does not specify any single state. 
The opposite direction, namely the derivation of solutions of (4) starting from solutions of (1) also can face severe mathematical difficulties in various points. In particular if we prescribe $\psi(0)$, we can define $\rho(0)$ and $J(0)$, however from the evolution of the quantities $\rho(t)$ and $J(t)$, we cannot reconstruct the wave function $\psi(t)$. Furthermore, from the moment equation in (1) we cannot derive the quantum eikonal equation

$$
\partial_{t} S+\frac{1}{2}|\nabla S|^{2}+h(\rho)+V+S=\frac{\hbar^{2}}{2} \frac{\Delta \sqrt{\rho}}{\sqrt{\rho}}
$$

which is the key element to reconstruct a solution of (4) via the WKB ansatz for the wave function, $\psi=\sqrt{\rho} e^{i S / \hbar}$.

Similar difficulties arise when approaching with Wigner functions, which has been recently quite popular to deduce quantum fluid systems in a kinetic way (see [54]). Even in the case we know the initial data $\rho(0), J(0)$ to be originated from a wave function $\psi(0)$, it is very difficult to show that the solutions $\rho(t), J(t)$ coincide for all times with the first and second momenta of the Wigner function obtained by solving the Wigner quantum transport equation. Moreover in our case there is also the difficulty due to the non-classical potential $\tilde{V}$.

A related question has been investigated by Bourgain, Brezis, Mironescu in several papers (see [6] and references therein) regarding the lifting problem for harmonic maps.

Another formal approach is provided by the following transport equation

$$
\rho \partial_{t} S+J \nabla S+\rho h(\rho)+\rho V+\rho S=\frac{\hbar^{2}}{2} \sqrt{\rho} \Delta \sqrt{\rho}
$$

which is obtained by multiplying equation (5) by $\rho$. Unfortunately the lack of regularity of the solutions does not allow to apply even the more recent advances of the theory of transport equations [16], [1] or the somehow related approach developed by Teufel and Tumulka [51] in the study of trajectories of Bohmian mechanics.

A related problem arises in the study of Nelson stochastic mechanics (see for instance Nelson [42], Guerra and Morato [24]), where the mathematical theory is based on the analysis of the velocity fields (see Carlen [7]). The application of this approach to our problem presents the same level of difficulty of the previously mentioned methods from the transport theory.

A natural framework to study the existence of the weak solutions to (1) is given by the space of finite energy states. Here the energy associated 
to the system (1) is given by

$$
E(t):=\int_{\mathbb{R}^{3}} \frac{\hbar^{2}}{2}|\nabla \sqrt{\rho(t)}|^{2}+\frac{1}{2}|\Lambda(t)|^{2}+f(\rho(t))+\frac{1}{2}|\nabla V(t)|^{2} \mathrm{~d} x,
$$

where $\Lambda:=J / \sqrt{\rho}$, and $f(\rho)=\frac{2}{p+1} \rho^{\frac{p+1}{2}}$. The function $f(\rho)$ denotes the internal energy, which is related to the pressure through the identity $P(\rho)=\rho f^{\prime}(\rho)-f(\rho)$.

Therefore our initial data are required to satisfy

$$
E_{0}:=\int_{\mathbb{R}^{3}} \frac{\hbar^{2}}{2}\left|\nabla \sqrt{\rho_{0}}\right|^{2}+\frac{1}{2}\left|\Lambda_{0}\right|^{2}+f\left(\rho_{0}\right)+\frac{1}{2}\left|\nabla V_{0}\right|^{2} \mathrm{~d} x<\infty,
$$

or equivalently (if we have $1 \leq p \leq 5$ ),

$$
\sqrt{\rho_{0}} \in H^{1}\left(\mathbb{R}^{3}\right) \quad \text { and } \quad \Lambda_{0}:=J_{0} / \sqrt{\rho_{0}} \in L^{2}\left(\mathbb{R}^{3}\right) .
$$

Definition 1. We say the pair $(\rho, J)$ is a weak solution of the Cauchy problem (1), (2) in $[0, T) \times \mathbb{R}^{3}$ with Cauchy data $\left(\rho_{0}, J_{0}\right) \in L^{2}\left(\mathbb{R}^{3}\right)$, if there exist locally integrable functions $\sqrt{\rho}, \Lambda$, such that $\sqrt{\rho} \in L_{l o c}^{2}\left([0, T) ; H_{l o c}^{1}\left(\mathbb{R}^{3}\right)\right)$, $\Lambda \in L_{l o c}^{2}\left([0, T) ; L_{l o c}^{2}\left(\mathbb{R}^{3}\right)\right)$ and by defining $\rho:=(\sqrt{\rho})^{2}, J:=\sqrt{\rho} \Lambda$, one has

- for any test function $\eta \in \mathcal{C}_{0}^{\infty}\left([0, T) \times \mathbb{R}^{3}\right)$ we have

$$
\int_{0}^{T} \int_{\mathbb{R}^{3}} \rho \partial_{t} \eta+J \cdot \nabla \eta \mathrm{d} x \mathrm{~d} t+\int_{\mathbb{R}^{3}} \rho_{0} \eta(0) \mathrm{d} x=0 ;
$$

- for any test function $\zeta \in \mathcal{C}_{0}^{\infty}\left([0, T) \times \mathbb{R}^{3} ; \mathbb{R}^{3}\right)$

$$
\begin{aligned}
& \int_{0}^{T} \int_{\mathbb{R}^{3}} J \cdot \partial_{t} \zeta+\Lambda \otimes \Lambda: \nabla \zeta+P(\rho) \operatorname{div} \zeta-\rho \nabla V \cdot \zeta-J \cdot \zeta \\
& +\hbar^{2} \nabla \sqrt{\rho} \otimes \nabla \sqrt{\rho}: \nabla \zeta-\frac{\hbar^{2}}{4} \rho \Delta \operatorname{div} \zeta \mathrm{d} x \mathrm{~d} t+\int_{\mathbb{R}^{3}} J_{0} \cdot \zeta(0) \mathrm{d} x=0 ;
\end{aligned}
$$

- (generalized irrotationality condition) for almost every $t \in(0, T)$,

$$
\nabla \wedge J=2 \nabla \sqrt{\rho} \wedge \Lambda
$$

holds in the sense of distributions.

Remark 2. Suppose we are in the smooth case, so that we can factorize $J=\rho u$, for some current velocity field $u$, then the last condition (12) simply means $\rho \nabla \wedge u=0$, the current velocity $u$ is irrotational in $\rho \mathrm{d} x$. This is why we will call it generalized irrotationality condition.

Definition 3. We say that the weak solution $(\rho, J)$ to the Cauchy problem (1), (2) is a finite energy weak solution (FEWS) in $[0, T) \times \mathbb{R}^{3}$, if in addition for almost every $t \in[0, T)$, the energy (7) is finite. 
In the sequel we restrict our attention only to FEWS with $\rho_{0} \in$ $L^{1}\left(\mathbb{R}^{3}\right)$. The hydrodynamic structure of the system $(1),(2)$ should not lead to conclude that the solutions behave like classical fluids. Indeed the connection with Schrödinger equations suggests that $\rho_{0}, J_{0}$ should in any case be seen as momenta related to some wave function $\psi_{0}$. The main result of this paper is the existence of FEWS by assuming the initial data $\rho_{0}, J_{0}$ are momenta of some wave function $\psi_{0} \in H^{1}\left(\mathbb{R}^{3}\right)$.

Theorem 4 (Main Theorem). Let $\psi_{0} \in H^{1}\left(\mathbb{R}^{3}\right)$ and let us define

$$
\rho_{0}:=\left|\psi_{0}\right|^{2}, \quad J_{0}:=\hbar \operatorname{Im}\left(\overline{\psi_{0}} \nabla \psi_{0}\right) .
$$

Then, for each $0<T<\infty$, there exists a finite energy weak solution to the $Q H D$ system $(1)$ in $[0, T) \times \mathbb{R}^{3}$, with initial data $\left(\rho_{0}, J_{0}\right)$ defined as above.

Remark 5. As we said above, we can extend the result of the previous main Theorem 4 to a more general type of collision term. Indeed it is possible to consider nonlinear terms of the type $f(\sqrt{\rho}, J, \nabla \sqrt{\rho})=$ $\alpha J+\rho \nabla g(t, x, \sqrt{\rho}, \Lambda, \nabla \sqrt{\rho})$, where $\alpha \geq 0$ and $g$ satisfies the following Carathèodory type conditions

- for all $(\sqrt{\rho}, \Lambda) \in[0, \infty) \times \mathbb{R}^{3}$, the function $(t, x) \mapsto g(t, x, \sqrt{\rho}, \Lambda, \nabla \sqrt{\rho})$ is Lebesgue measurable;

- for almost all $(t, x) \in[0, \infty) \times \mathbb{R}^{3}$, the function $(u, v, w) \mapsto$ $g(t, x, u, v, w)$ is continuous for $(u, v, w) \in[0, \infty) \times \mathbb{R}^{3} \times \mathbb{R}^{3}$ :

- there exists $C \in L^{\infty}\left([0, \infty) \times \mathbb{R}^{3}\right)$ such that

$$
|g(t, x, u, v, w)| \leq C(t, x)\left(1+|u|^{4}+|v|^{4 / 3}+|w|^{4 / 3}\right) .
$$

The case $\alpha=0$ requires a slight modification of the method presented in the Section 4, as we are going to explain below. The case $\alpha>0$ can be treated with minor modifications of the finite difference scheme in the Section 5 defined for $f(\sqrt{\rho}, J, \nabla \sqrt{\rho})=J$. See the Remark 22 below in the section 5.

We remark that the condition (13) is needed in order to ensure $g \in$ $L^{\infty}\left([0, \infty) ; L^{p}\left(\mathbb{R}^{3}\right)+L^{\infty}\left(\mathbb{R}^{3}\right)\right)$ as long as $\sqrt{\rho} \in L^{\infty}\left([0, T) ; H^{1}\left(\mathbb{R}^{3}\right)\right)$, $\Lambda \in L^{\infty}\left([0, T) ; L^{2}\left(\mathbb{R}^{3}\right)\right)$. Note that with this definition of $f$ we need to change the definition given before for the weak solutions. Indeed the balance law for the current density becomes

$$
\begin{aligned}
\int_{0}^{\infty} \int_{\mathbb{R}^{3}} J \cdot \partial_{t} \zeta+ & \Lambda \otimes \Lambda: \nabla \zeta+P(\rho) \operatorname{div} \zeta-\rho \nabla V \cdot \zeta-J \cdot \zeta \\
& +g(t, x, \sqrt{\rho}, \Lambda)(\nabla \rho \cdot \zeta+\rho \operatorname{div} \zeta) \\
+ & \hbar^{2} \nabla \sqrt{\rho} \otimes \nabla \sqrt{\rho}: \nabla \zeta-\frac{\hbar^{2}}{4} \rho \Delta \operatorname{div} \zeta \mathrm{d} x \mathrm{~d} t+\int_{\mathbb{R}^{3}} J_{0} \cdot \zeta(0) \mathrm{d} x=0 .
\end{aligned}
$$


In the Sections 5 and 6 we will explain how to modify our methods to include this case (see Remarks 22 and 29).

At the end of this Section we would like to remark that the system (1) is closely related to the Quantum Drift-Diffusion (QDD) equation

$$
\partial_{t} \rho+\operatorname{div}\left(\rho \nabla\left(\frac{\hbar^{2}}{2} \frac{\Delta \sqrt{\rho}}{\rho}-V-h(\rho)\right)\right),
$$

where $V$ is again the electrostatic potential and the enthalpy $h(\rho)$ is such that $\rho h^{\prime}(\rho)=P^{\prime}(\rho)$. Indeed if we scale the collision term $J$ in (1) with a relaxation time $\varepsilon$ and we write

$$
\left\{\begin{array}{l}
\partial_{t} \rho^{\varepsilon}+\operatorname{div} J^{\varepsilon}=0 \\
\partial_{t} J^{\varepsilon}+\operatorname{div}\left(\frac{J^{\varepsilon} \otimes J^{\varepsilon}}{\rho^{\varepsilon}}\right)+\nabla P\left(\rho^{\varepsilon}\right)+\rho^{\varepsilon} \nabla V^{\varepsilon}+\frac{1}{\varepsilon} J^{\varepsilon}=\frac{\hbar^{2}}{2} \rho^{\varepsilon} \nabla\left(\frac{\Delta \sqrt{\rho^{\varepsilon}}}{\sqrt{\rho^{\varepsilon}}}\right) \\
-\Delta V^{\varepsilon}=\rho^{\varepsilon}-C(x),
\end{array}\right.
$$

then in the formal limit $\varepsilon \rightarrow 0$ we get the equation (14). A partial result on this limit was given by Jüngel, Li and Matsumura in [26], while in [22] it is shown the global existence of non-negative variational solutions to (14) without the electrostatic potential and the pressure term. In a forthcoming paper we deal with the relaxation limit from solutions to (15) to solutions of (14).

\section{Preliminaries and Notations}

2.1. Notations. For convenience of the reader we will set some notations which will be used in the sequel.

If $X, Y$ are two quantities (typically non-negative), we use $X \lesssim Y$ to denote $X \leq C Y$, for some absolute constant $C>0$.

We will use the standard Lebesgue norms for complex-valued measurable functions $f: \mathbb{R}^{d} \rightarrow \mathbb{C}$

$$
\|f\|_{L^{p}\left(\mathbb{R}^{d}\right)}:=\left(\int_{\mathbb{R}^{d}}|f(x)|^{p} \mathrm{~d} x\right)^{\frac{1}{p}} .
$$

If we replace $\mathbb{C}$ by a Banach space $X$, we will adopt the notation

$$
\|f\|_{L^{p}\left(\mathbb{R}^{d} ; X\right)}:=\left(\int_{\mathbb{R}^{d}}\|f(x)\|_{X} \mathrm{~d} x\right)^{1 / p}
$$

to denote the norm of $f: \mathbb{R}^{d} \rightarrow X$. In particular, if $\mathrm{X}$ is a Lebesgue space $L^{r}\left(\mathbb{R}^{n}\right)$, and $d=1$, we will shorten the notation by writing

$$
\|f\|_{L_{t}^{q} L_{x}^{r}\left(I \times \mathbb{R}^{n}\right)}:=\left(\int_{I}\|f(t)\|_{L^{r}\left(\mathbb{R}^{n}\right)}^{q} \mathrm{~d} t\right)^{1 / q}=\left(\int_{I}\left(\int_{\mathbb{R}^{n}}|f(t, x)|^{r} \mathrm{~d} x\right)^{q / r} \mathrm{~d} t\right)^{1 / q}
$$


to denote the mixed Lebesgue norm of $f: I \rightarrow L^{r}\left(\mathbb{R}^{n}\right)$; moreover, we will write $L_{t}^{q} L_{x}^{r}\left(I \times \mathbb{R}^{n}\right):=L^{q}\left(I ; L^{r}\left(\mathbb{R}^{n}\right)\right)$.

For $s \in \mathbb{R}$ we will define the Sobolev space $H^{s}\left(\mathbb{R}^{n}\right):=(1-\Delta)^{-s / 2} L^{2}\left(\mathbb{R}^{n}\right)$.

Definition 6 (see [8]). We say that $(q, r)$ is an admissible pair of exponents if $2 \leq q \leq \infty, 2 \leq r \leq 6$, and

$$
\frac{1}{q}=\frac{3}{2}\left(\frac{1}{2}-\frac{1}{r}\right) \text {. }
$$

Now we will introduce the Strichartz norms. For more details, we refer the reader to [50], [11]. Let $I \times \mathbb{R}^{3}$ be a space-time slab, we define the Strichartz norm $\dot{S}^{0}\left(I \times \mathbb{R}^{3}\right)$

$$
\|u\|_{\dot{S}^{0}\left(I \times \mathbb{R}^{3}\right)}:=\sup \left(\sum_{N}\left\|P_{N} u\right\|_{L_{t}^{q} L_{x}^{r}\left(I \times \mathbb{R}^{3}\right)}^{2}\right)^{1 / 2}
$$

where the sup is taken over all the admissible pairs $(q, r)$. Here $P_{N}$ denotes the Paley-Littlewood projection operator, with the sum taken over diadic numbers of the form $N=2^{j}, j \in \mathbb{Z}$. For any $k \geq 1$, we can define

$$
\|u\|_{\dot{S}^{k}\left(I \times \mathbb{R}^{3}\right)}:=\left\|\nabla^{k} u\right\|_{\dot{S}^{0}\left(I \times \mathbb{R}^{3}\right)} .
$$

Note that, from Paley-Littlewood inequality we have

$\|u\|_{L_{t}^{q} L_{x}^{r}\left(I \times \mathbb{R}^{3}\right)} \lesssim\left\|\left(\sum_{N}\left|P_{N} u\right|^{2}\right)^{1 / 2}\right\|_{L_{t}^{q} L_{x}^{r}\left(I \times \mathbb{R}^{3}\right)} \lesssim\left(\sum_{N}\left\|P_{N} u\right\|_{L_{t}^{q} L_{x}^{r}\left(I \times \mathbb{R}^{3}\right)}^{2}\right)^{1 / 2}$,

and hence for each admissible pair of exponents, one has

$$
\|u\|_{L_{t}^{q} L_{x}^{r}\left(I \times \mathbb{R}^{n}\right)} \lesssim\|u\|_{\dot{S}^{0}\left(I \times \mathbb{R}^{3}\right)}
$$

Lemma 7 ([11]).

$$
\|u\|_{L_{t}^{4} L_{x}^{\infty}\left(I \times \mathbb{R}^{3}\right)} \lesssim\|u\|_{\dot{S}^{1}\left(I \times \mathbb{R}^{3}\right)} .
$$

2.2. Schrödinger equations. In this paragraph we recall some important results concerning the nonlinear Schrödinger equations that will be used throughout the paper. First of all, we recall a global wellposedness theorem for the nonlinear Schrödinger-Poisson system (see [8] and the references therein):

Theorem 8. Let us consider the following nonlinear Schrödinger-Poisson system

$$
\left\{\begin{array}{l}
i \hbar \partial_{t} \psi+\frac{\hbar^{2}}{2} \Delta \psi=|\psi|^{p-1} \psi+V \psi \\
-\Delta V=|\psi|^{2}
\end{array}\right.
$$


with the initial datum

$$
\psi(0)=\psi_{0} \in H^{1}\left(\mathbb{R}^{3}\right) .
$$

There exists a unique globally defined strong solution $\psi \in \mathcal{C}\left(\mathbb{R} ; H^{1}\left(\mathbb{R}^{3}\right)\right)$, which depends continuously on the initial data. Furthermore, the total energy

$$
E(t):=\int_{\mathbb{R}^{3}} \frac{\hbar^{2}}{2}|\nabla \psi(t, x)|^{2}+\frac{2}{p+1}|\psi(t, x)|^{p+1}+V(t, x)|\psi(t, x)|^{2} \mathrm{~d} x
$$

is conserved, namely

$$
E(t)=E_{0}, \quad \text { for all } t \in \mathbb{R} .
$$

Moreover, the dispersive nature of Schrödinger equation provides also some further integrability and regularity properties of the solutions. The first result in this direction regards some space-time integrability properties, the most important of them are the well known Strichartz estimates for the Schrödinger equation in $\mathbb{R}^{1+3}$. We refer to the classical paper of Ginibre and Velo [23], the paper of Keel and Tao [33], the books of Cazenave [8] and Tao [50] and the references therein. Let $\psi$ is the unique (strong) solution of the Cauchy problem for the free Schrödinger equation

$$
\left\{\begin{array}{l}
i \partial_{t} \psi+\Delta \psi=0 \\
\psi(0)=\psi_{0}
\end{array}\right.
$$

in the following we shalll denote by $U(\cdot)$ the free Schrödinger group, defined by the indentity $U(t) \psi_{0}=\psi(t)$.

The next result is taken from [33] and the estimates are obtained in a very general setting.

Theorem 9 (Keel, Tao [33]). Let $(q, r),(\tilde{q}, \tilde{r})$ be two arbitrary admissible pairs of exponents, and let $U(\cdot)$ be the free Schrödinger group. Then we have

$$
\begin{aligned}
&\|U(t) f\|_{L_{t}^{q} L_{x}^{r}} \lesssim\|f\|_{L^{2}\left(\mathbb{R}^{3}\right)} \\
&\left\|\int_{s<t} U(t-s) F(s) \mathrm{d} s\right\|_{L_{t}^{q} L_{x}^{r}} \lesssim\|F\|_{L_{t}^{\tilde{q}^{\prime}} L_{x}^{\tilde{r}^{\prime}}} \\
&\left\|\int U(t-s) F(s) \mathrm{d} s\right\|_{L_{t}^{q} L_{x}^{r}} \lesssim\|F\|_{L_{t}^{\tilde{q}^{\prime}} L_{x}^{\tilde{r}^{\prime}}}
\end{aligned}
$$

By using the previous Theorem, we can state the following Lemma about some further integrability properties for solutions of the Schrödinger equations. 
Lemma 10 ([11]). Let $I$ be a compact interval, and let $u: I \times \mathbb{R}^{3} \rightarrow \mathbb{C}$ be a Schwartz solution to the Schrödinger equation

$$
i \partial_{t} u+\Delta u=F_{1}+\ldots+F_{M}
$$

for some Schwartz functions $F_{1}, \ldots, F_{M}$. Then we have

$\|u\|_{\dot{S}^{0}\left(I \times \mathbb{R}^{n}\right)} \lesssim\left\|u\left(t_{0}\right)\right\|_{L^{2}\left(\mathbb{R}^{n}\right)}+\left\|F_{1}\right\|_{L_{t}^{q_{1}^{\prime}} L_{x}^{r_{1}^{\prime}\left(I \times \mathbb{R}^{3}\right)}}+\ldots+\left\|F_{M}\right\|_{L_{t}^{q_{M}^{\prime}} L_{x}^{r_{M}^{\prime}\left(I \times \mathbb{R}^{3}\right)}}$, where $\left(q_{1}, r_{1}\right), \ldots,\left(q_{M}, r_{M}\right)$ are arbitrary admissible pairs of exponents.

Furthermore, the solutions to the Schrödinger equation enjoy some nice local smoothing properties; there are various results in the literature regarding this property, here we recall a theorem, due to Constatin and Saut [9] which actually covers a more general setting. In particular it can been shown the solutions of the Schrödinger equation are locally more regular than their initial data. Close results have been proved by P. Sjölin [48] and L. Vega [52].

Theorem 11 (Constantin, Saut [9]). Let u solves the free Schrödinger equation

$$
\left\{\begin{array}{l}
i \partial_{t} u+\Delta u=0 \\
u(0)=u_{0}
\end{array}\right.
$$

Let $\chi \in \mathcal{C}_{0}^{\infty}\left(\mathbb{R}^{1+3}\right)$ of the form

$$
\chi(t, x)=\chi_{0}(t) \chi_{1}\left(x_{1}\right) \chi_{2}\left(x_{2}\right) \chi\left(x_{3}\right),
$$

with $\chi_{j} \in \mathcal{C}_{0}^{\infty}(\mathbb{R})$. Then we have

$$
\int_{\mathbb{R}^{1+3}} \chi^{2}(t, x)\left|(I-\Delta)^{1 / 4} u(t, x)\right|^{2} \mathrm{~d} x \mathrm{~d} t \leq C^{2}\left\|u_{0}\right\|_{L^{2} \mathbb{R}^{3}} .
$$

In particular, if $u_{0} \in L^{2}\left(\mathbb{R}^{3}\right)$, one has for all $T>0$

$$
u \in L^{2}\left([0, T] ; H_{l o c}^{1 / 2}\left(\mathbb{R}^{3}\right)\right) .
$$

We have a similar result also for the nonhomogeneous case:

Theorem 12 (Constantin, Saut [9]). Let $u$ be the solution of

$$
\left\{\begin{array}{l}
i \partial_{t} u+\Delta u=F \\
u(0)=u_{0} \in L^{2}\left(\mathbb{R}^{3}\right),
\end{array}\right.
$$

where $F \in L^{1}\left([0, T] ; L^{2}\left(\mathbb{R}^{3}\right)\right)$. Then it follows

$$
u \in L^{2}\left([0, T] ; H_{l o c}^{1 / 2}\left(\mathbb{R}^{3}\right)\right) .
$$

Moreover, let $\chi \in \mathcal{C}_{0}^{\infty}\left(\mathbb{R}^{1+3}\right)$ be of the form

$$
\chi(t, x)=\chi_{0}(t) \chi_{1}\left(x_{1}\right) \chi_{2}\left(x_{2}\right) \chi_{3}\left(x_{3}\right)
$$


with $\chi_{j} \in \mathcal{C}_{0}^{\infty}(\mathbb{R})$, supp $\chi_{0} \subset[0, T]$. Then the following local smoothing estimate holds

$$
\begin{aligned}
& \left(\int_{\mathbb{R}^{1+3}} \chi^{2}(t, x)\left|(I-\Delta)^{1 / 4} u(t, x)\right|^{2} \mathrm{~d} x \mathrm{~d} t\right)^{\frac{1}{2}} \\
& \leq C\left(\left\|u_{0}\right\|_{L^{2}\left(\mathbb{R}^{3}\right)}+\|F\|_{L_{t}^{1} L_{x}^{2}\left([0, T] \times \mathbb{R}^{3}\right)}\right)
\end{aligned}
$$

This results imply that, the free Schrödinger group $U(\cdot)$ fulfills the following inequalities

$$
\begin{gathered}
\left\|U(\cdot) u_{0}\right\|_{L^{2}\left([0, T] ; H_{l o c}^{1 / 2}\right)} \lesssim\left\|u_{0}\right\|_{L^{2}\left(\mathbb{R}^{3}\right)} \\
\left\|\int_{0}^{t} U(t-s) F(s) \mathrm{d} s\right\|_{L^{2}\left([0, T] ; H_{l o c}^{1 / 2}\left(\mathbb{R}^{3}\right)\right)} \lesssim\|F\|_{L^{1}\left([0, T] ; L^{2}\left(\mathbb{R}^{3}\right)\right)} .
\end{gathered}
$$

2.3. Compactness tools. Here we recall some compactness theorems in function spaces, which will be relevant in the Section 6 to prove the convergence of the approximate solutions. Let us recall in particular a compactness result due to Rakotoson, Temam [44] in the spirit of classical results of Aubin, Lions and Simon, see [47], [3], [39].

Theorem 13 (Rakotoson, Temam [44]). Let $\left(V,\|\cdot\|_{V}\right),\left(H ;\|\cdot\|_{H}\right)$ be two separable Hilbert spaces. Assume that $V \subset H$ with a compact and dense embedding. Consider a sequence $\left\{u^{\varepsilon}\right\}$, converging weakly to a function $u$ in $L^{2}([0, T] ; V), T<\infty$. Then $u^{\varepsilon}$ converges strongly to $u$ in $L^{2}([0, T] ; H)$, if and only if

(1) $u^{\varepsilon}(t)$ converges to $u(t)$ weakly in $H$ for a.e. $t$;

(2) $\lim _{|E| \rightarrow 0, E \subset[0, T]} \sup _{\varepsilon>0} \int_{E}\left\|u^{\varepsilon}(t)\right\|_{H}^{2} \mathrm{~d} t=0$.

\section{Polar Decomposition}

In this section we will explain how to decompose an arbitrary wave function $\psi$ into its amplitude $\sqrt{\rho}=|\psi|$ and its unitary factor $\phi$, namely a function taking its values in the unitary circle of the complex plane, such that $\psi=\sqrt{\rho} \phi$. The idea is similar in the spirit, to that one used by Y. Brenier in [5], to find the measure preserving maps needed to write vector-valued functions as compositions of gradients of convex functions and measure preserving maps. Our case is much simpler than [5] and it can be studied directly in a simpler setting. Brenier's idea looks for projections of $L^{2}$ functions $u$ onto the set of measure preserving maps $S$, contained in a given sphere of $L^{2}$, i.e. one has to find $s \in S$, which minimizes the distance $\|u-s\|_{L^{2}}$, or equivalently, which maximizes $(u, s)_{L^{2}}$. In our case we should maximize $\operatorname{Re}(u, s)_{L^{2}}$, within complex-valued functions with the constraint to take value in the unit ball of $L^{\infty}$. Actually in this case the maximizer turns out to 
be trivially determined and the previous variational argument has only to be considered a motivation to the subsequent considerations.

However in the case we assume $u \in H^{1}\left(\mathbb{R}^{3}\right)$ (that is the relevant situation in physics), then it would be useful to know the regularity of the maximizers in the light of a possible use of the considerations explained in the Remark 27.

Let us consider a wave function $\psi \in L^{2}\left(\mathbb{R}^{3}\right)$ and define the set (29)

$$
P(\psi):=\left\{\phi \text { measurable } \mid\|\phi\|_{L^{\infty}\left(\mathbb{R}^{3}\right)} \leq 1, \sqrt{\rho} \phi=\psi \text { a. e. in } \mathbb{R}^{3}\right\},
$$

where $\sqrt{\rho}=|\psi|$. Of course if we consider $\phi \in P(\psi)$, then by the definition of the set $P(\psi)$ it is immediate that $|\phi|=1$ a.e. $\sqrt{\rho}-\mathrm{d} x$ in $\mathbb{R}^{3}$ and $\phi \in P(\psi)$ is uniquely determined a.e. $\sqrt{\rho}-\mathrm{d} x$ in $\mathbb{R}^{3}$.

Remark 14. Let $B_{R}$ be the ball in $\mathbb{R}^{3}$ with radius $R$, centered at the origin and let us define the set

$$
P_{R}(\psi):=\left\{\phi \text { measurable } \mid\|\phi\|_{L^{\infty}\left(\mathbb{R}^{3}\right)} \leq 1, \sqrt{\rho} \phi=\psi \text { a. e. in } \mathbb{R}^{3}\right\} \text {. }
$$

Then it is easy to see that $\phi \in P_{R}(\psi)$ if and only if $\phi$ is a maximizer for the functional

$$
\Phi_{R}[\phi]:=\operatorname{Re} \int_{B_{R}} \bar{\psi} \phi \mathrm{d} x
$$

over the set

$$
S_{R}:=\left\{\phi \in L^{2}\left(B_{R}\right) \mid\|\phi\|_{L^{\infty}\left(B_{R}\right)} \leq 1\right\} .
$$

The next lemma connects the structure of the bilinear term $\hbar^{2} \operatorname{Re}(\nabla \overline{\psi \otimes \nabla \psi})$ with $\nabla \sqrt{\rho} \otimes \nabla \sqrt{\rho}$ and $\frac{J \otimes J}{\rho}$. Moreover it shows this structure is $H^{1}$ stable.

Lemma 15. Let $\psi \in H^{1}\left(\mathbb{R}^{3}\right), \sqrt{\rho}:=|\psi|$, then there exists $\phi \in L^{\infty}\left(\mathbb{R}^{3}\right)$ such that $\psi=\sqrt{\rho} \phi$ a.e. in $\mathbb{R}^{3}, \sqrt{\rho} \in H^{1}\left(\mathbb{R}^{3}\right), \nabla \sqrt{\rho}=\operatorname{Re}(\bar{\phi} \nabla \psi)$. If we set $\Lambda:=\hbar \operatorname{Im}(\bar{\phi} \nabla \psi)$, one has $\Lambda \in L^{2}\left(\mathbb{R}^{3}\right)$ and moreover the following identity holds

$$
\hbar^{2} \operatorname{Re}\left(\partial_{j} \bar{\psi} \partial_{k} \psi\right)=\hbar^{2} \partial_{j} \sqrt{\rho} \partial_{k} \sqrt{\rho}+\Lambda^{(j)} \Lambda^{(k)} .
$$

Furthermore, let $\psi_{n} \rightarrow \psi$ strongly in $H^{1}\left(\mathbb{R}^{3}\right)$, then it follows

$$
\nabla \sqrt{\rho_{n}} \rightarrow \nabla \sqrt{\rho}, \quad \Lambda_{n} \rightarrow \Lambda \quad \text { in } L^{2}\left(\mathbb{R}^{3}\right),
$$

where $\Lambda_{n}:=\hbar \operatorname{Im}\left(\overline{\phi_{n}} \nabla \psi_{n}\right)$.

Proof. Let us consider a sequence $\left\{\psi_{n}\right\} \subset \mathcal{C}^{\infty}\left(\mathbb{R}^{3}\right), \psi \rightarrow \psi$ in $H^{1}\left(\mathbb{R}^{3}\right)$, define as before

$$
\phi_{n}(x)=\left\{\begin{aligned}
\frac{\psi_{n}(x)}{\left|\psi_{n}(x)\right|} & \text { if } \psi_{n}(x) \neq 0 \\
0 & \text { if } \psi(x)=0 .
\end{aligned}\right.
$$


Then, there exists $\phi \in L^{\infty}\left(\mathbb{R}^{3}\right)$ such that $\phi_{n} \stackrel{*}{\rightarrow} \phi$ in $L^{\infty}\left(\mathbb{R}^{3}\right)$ and $\nabla \psi_{n} \rightarrow \nabla \psi$ in $L^{2}\left(\mathbb{R}^{3}\right)$, hence

$$
\operatorname{Re}\left(\overline{\phi_{n}} \nabla \psi_{n}\right) \rightarrow \operatorname{Re}(\bar{\phi} \nabla \psi) \quad \text { in } L^{2}\left(\mathbb{R}^{3}\right) .
$$

Since by (32), one has

$$
\operatorname{Re}\left(\overline{\phi_{n}} \nabla \psi_{n}\right)=\nabla\left|\psi_{n}\right|
$$

a.e. in $\mathbb{R}^{3}$, it follows

$$
\nabla \sqrt{\rho_{n}} \rightarrow \operatorname{Re}(\bar{\phi} \nabla \psi) \quad \text { in } L^{2}\left(\mathbb{R}^{3}\right) .
$$

Moreover, one has $\nabla \sqrt{\rho_{n}} \rightarrow \nabla \sqrt{\rho}$ in $L^{2}\left(\mathbb{R}^{3}\right)$, therefore

$$
\nabla \sqrt{\rho}=\operatorname{Re}(\bar{\phi} \nabla \psi)
$$

where $\phi$ is a unitary factor of $\psi$.

The identity (30) follows immediately fro the following

$$
\begin{aligned}
\hbar^{2} \operatorname{Re}\left(\partial_{j} \bar{\psi} \partial_{k} \psi\right)= & \hbar^{2} \operatorname{Re}\left(\left(\phi \partial_{j} \bar{\psi}\right)\left(\bar{\phi} \partial_{k} \psi\right)\right)=\hbar^{2} \operatorname{Re}\left(\phi \partial_{j} \bar{\phi}\right) \operatorname{Re}\left(\bar{\phi} \partial_{k} \psi\right) \\
& -\hbar^{2} \operatorname{Im}\left(\phi \partial_{j} \bar{\psi}\right) \operatorname{Im}\left(\bar{\phi} \partial_{k} \psi\right)=\hbar^{2} \partial_{j} \sqrt{\rho} \partial_{k} \sqrt{\rho}+\Lambda^{(j)} \Lambda^{(k)} .
\end{aligned}
$$

Now we prove (31). Let us take a sequence $\psi_{n} \rightarrow \psi$ strongly in $H^{1}\left(\mathbb{R}^{3}\right)$, and consider $\nabla \sqrt{\rho_{n}}=\operatorname{Re}\left(\overline{\phi_{n}} \nabla \psi_{n}\right), \Lambda_{n}:=\hbar \operatorname{Im}\left(\overline{\phi_{n}} \nabla \psi_{n}\right)$. As before, $\phi_{n} \stackrel{*}{\rightarrow} \phi$ in $L^{\infty}\left(\mathbb{R}^{3}\right)$, with $\phi$ a polar factor for $\psi$; then $\nabla \sqrt{\rho_{n}} \rightarrow \nabla \sqrt{\rho}$, $\operatorname{Re}\left(\overline{\phi_{n}} \nabla \psi_{n}\right) \rightarrow \operatorname{Re}(\bar{\phi} \nabla \psi)$, and $\nabla \sqrt{\rho}=\operatorname{Re}(\bar{\phi} \nabla \psi)$. Moreover, $\Lambda_{n}:=$ $\hbar \operatorname{Im}\left(\overline{\phi_{n}} \nabla \psi_{n}\right) \rightarrow \hbar \operatorname{Im}(\bar{\phi} \nabla \psi)=: \Lambda$. To upgrade the weak convergence into the strong one, simply notice that by (30) one has

$$
\begin{array}{r}
\hbar^{2}\|\nabla \psi\|_{L^{2}}^{2}=\hbar^{2}\|\nabla \sqrt{\rho}\|_{L^{2}}^{2}+\|\Lambda\|_{L^{2}}^{2} \leq \liminf _{n \rightarrow \infty}\left(\hbar^{2}\left\|\nabla \sqrt{\rho_{n}}\right\|_{L^{2}}^{2}+\left\|\Lambda_{n}\right\|_{L^{2}}^{2}\right) \\
=\liminf _{n \rightarrow \infty} \hbar^{2}\left\|\nabla \psi_{n}\right\|_{L^{2}}^{2}=\hbar^{2}\|\nabla \psi\|_{L^{2}}^{2}
\end{array}
$$

Corollary 16. Let $\psi \in H^{1}\left(\mathbb{R}^{3}\right)$, then

$$
\hbar \nabla \bar{\psi} \wedge \nabla \psi=2 i \nabla \sqrt{\rho} \wedge \Lambda
$$

Proof. It suffices to note that we can write

$$
\hbar \nabla \bar{\psi} \wedge \nabla \psi=\hbar(\phi \nabla \bar{\psi}) \wedge(\bar{\phi} \nabla \psi)
$$

where $\phi \in L^{\infty}\left(\mathbb{R}^{3}\right)$ is the polar factor of $\psi$ such that $\nabla \sqrt{\rho}=\operatorname{Re}(\bar{\phi} \nabla \psi)$, $\Lambda:=\hbar \operatorname{Im}(\bar{\phi} \nabla \psi)$, as in the Lemma 15 . By splitting $\bar{\phi} \nabla \psi$ into its real and imaginary part, we get the identity (33). 
Now we state a technical lemma which will be used in the next sections. It summarizes the results of this section we use later on and will be handy for the application to the fractional step method.

Lemma 17. Let $\psi \in H^{1}\left(\mathbb{R}^{3}\right)$, and let $\tau, \varepsilon>0$ be two arbitrary (small) real numbers. Then there exists $\tilde{\psi} \in H^{1}\left(\mathbb{R}^{3}\right)$ such that

$$
\begin{aligned}
& \tilde{\rho}=\rho \\
& \tilde{\Lambda}=(1-\tau) \Lambda+r_{\varepsilon},
\end{aligned}
$$

where $\sqrt{\rho}:=|\psi|, \sqrt{\tilde{\rho}}:=|\tilde{\psi}|, \Lambda:=\hbar \operatorname{Im}(\bar{\phi} \nabla \psi), \tilde{\Lambda}:=\hbar \operatorname{Im}(\overline{\tilde{\phi}} \nabla \tilde{\psi}), \phi, \tilde{\phi}$ are polar factors for $\psi, \tilde{\psi}$, respectively, and

$$
\left\|r_{\varepsilon}\right\|_{L^{2}\left(\mathbb{R}^{3}\right)} \leq \varepsilon
$$

Furthermore we have

$$
\nabla \tilde{\psi}=\nabla \psi-i \frac{\tau}{\hbar} \phi^{\star} \Lambda+r_{\varepsilon, \tau}
$$

where $\left\|\phi^{\star}\right\|_{L^{\infty}\left(\mathbb{R}^{3}\right)} \leq 1$ and

$$
\left\|r_{\varepsilon, \tau}\right\|_{L^{2}\left(\mathbb{R}^{3}\right)} \leq C\left(\tau\|\nabla \psi\|_{L^{2}\left(\mathbb{R}^{3}\right)}+\varepsilon\right) .
$$

Proof. Consider a sequence $\left\{\psi_{n}\right\} \subset \mathcal{C}^{\infty}\left(\mathbb{R}^{3}\right)$ converging to $\psi$ in $H^{1}\left(\mathbb{R}^{3}\right)$, and define

$$
\phi_{n}(x):= \begin{cases}\frac{\psi_{n}(x)}{\left|\psi_{n}(x)\right|} & \text { if } \psi_{n}(x) \neq 0 \\ 0 & \text { if } \psi_{n}(x)=0\end{cases}
$$

as polar factors for the wave functions $\psi_{n}$. Since $\psi_{n} \in \mathcal{C}^{\infty}$, then $\phi_{n}$ is piecewise smooth, and $\Omega_{n}:=\left\{x \in \mathbb{R}^{3}:\left|\psi_{n}(x)\right|>0\right\}$ is an open set, with smooth boundary. Therefore we can say there exists a function $\theta_{n}: \Omega_{n} \rightarrow[0,2 \pi)$, piecewise smooth in $\Omega_{n}$ and

$$
\phi_{n}(x)=e^{i \theta_{n}(x)}, \quad \text { for any } x \in \Omega_{n} .
$$

Moreover, by the previous Lemma, we have $\phi_{n} \stackrel{*}{\rightarrow} \phi$ in $L^{\infty}\left(\mathbb{R}^{3}\right)$, where $\phi$ is a polar factor of $\psi$, and $\Lambda_{n}:=\hbar \operatorname{Im}\left(\overline{\phi_{n}} \nabla \psi_{n}\right) \rightarrow \Lambda:=\hbar \operatorname{Im}(\bar{\phi} \nabla \psi)$ in $L^{2}\left(\mathbb{R}^{3}\right)$. Thus there exists $n \in \mathbb{N}$ such that

$$
\left\|\psi-\psi_{n}\right\|_{H^{1}\left(\mathbb{R}^{3}\right)}+\left\|\Lambda-\Lambda_{n}\right\|_{L^{2}\left(\mathbb{R}^{3}\right)} \leq \varepsilon .
$$

Now we can define

$$
\tilde{\psi}:=e^{i(1-\tau) \theta_{n}} \sqrt{\rho_{n}} .
$$

Let us remark that (36) is a good definition for $\tilde{\psi}$, since outside $\Omega_{n}$, where $\theta_{n}$ is not defined, we have $\sqrt{\rho_{n}}=0$, and hence also $\psi_{n}=0$. 
Furthermore,

$$
\begin{aligned}
\nabla \tilde{\psi}= & e^{-i \tau \theta_{n}} \nabla \psi_{n}-i \frac{\tau}{\hbar} e^{i(1-\tau) \theta_{n}} \Lambda_{n} \\
= & \nabla \psi-i \frac{\tau}{\hbar} e^{i(1-\tau) \theta_{n}} \Lambda_{n}+\tau\left(\sum_{j=1}^{\infty} \frac{\left(-i \theta_{n}\right)^{j} \tau^{j-1}}{j !}\right) \nabla \psi_{n} \\
& +\left(\nabla \psi_{n}-\nabla \psi\right)-i \frac{\tau}{\hbar} e^{i(1-\tau) \theta_{n}}\left(\Lambda_{n}-\Lambda\right)
\end{aligned}
$$

and thus we obtain (34) and (35), with $r_{\varepsilon, \tau}$ given by

$r_{\varepsilon, \tau}=\tau\left(\sum_{j=1}^{\infty} \frac{\left(-i \theta_{n}\right)^{j} \tau^{j-1}}{j !}\right) \nabla \psi_{n}+\left(\nabla \psi_{n}-\nabla \psi\right)-i \frac{\tau}{\hbar} e^{i(1-\tau) \theta_{n}}\left(\Lambda_{n}-\Lambda\right)$

Moreover

$$
\tilde{\Lambda}=\hbar \operatorname{Im}\left(e^{-i(1-\tau) \theta_{n}} \nabla \tilde{\psi}\right)=(1-\tau) \Lambda_{n}=(1-\tau) \Lambda+(1-\tau)\left(\Lambda_{n}-\Lambda\right)
$$

and clearly $r_{\varepsilon}:=(1-\tau)\left(\Lambda_{n}-\Lambda\right)$ has $L^{2}$-norm less than $\varepsilon$ by assumption.

\section{QHD WITHOUT COLLISIONS}

Now let us summarize some key points regarding the existence of weak solutions to the Quantum Hydrodynamic system, in the collisionless case.

The balance equations can be written in the following way:

$$
\left\{\begin{array}{l}
\partial_{t} \rho+\operatorname{div} J=0 \\
\partial_{t} J+\operatorname{div}\left(\frac{J \otimes J}{\rho}\right)+\nabla P(\rho)+\rho \nabla V=\frac{\hbar^{2}}{2} \rho \nabla\left(\frac{\Delta \sqrt{\rho}}{\sqrt{\rho}}\right) \\
-\Delta V=\rho,
\end{array}\right.
$$

where $P(\rho)=\frac{p-1}{p+1} \rho^{(p+1) / 2}, 1 \leq p<5$.

Definition 18. We say the pair $(\rho, J)$ is a weak solution in $[0, T) \times$ $\mathbb{R}^{3}$ to the system (38) with Cauchy data $\left(\rho_{0}, J_{0}\right) \in L_{l o c}^{1}\left(\mathbb{R}^{3}\right)$ if and only if there exist locally integrable functions $\sqrt{\rho}, \Lambda$ such that $\sqrt{\rho} \in$ $L_{\text {loc }}^{2}\left([0, T) ; H_{\text {loc }}^{1}\left(\mathbb{R}^{3}\right)\right), \Lambda \in L_{\text {loc }}^{2}\left([0, T) ; L_{\text {loc }}^{2}\left(\mathbb{R}^{3}\right)\right)$ and if we define $\rho:=$ $(\sqrt{\rho})^{2}, J:=\sqrt{\rho} \Lambda$, then

- for any test function $\varphi \in \mathcal{C}_{0}^{\infty}\left([0, T) \times \mathbb{R}^{3}\right)$ we have

$$
\int_{0}^{T} \int_{\mathbb{R}^{3}} \rho \partial_{t} \varphi+J \cdot \nabla \varphi \mathrm{d} x \mathrm{~d} t+\int_{\mathbb{R}^{3}} \rho_{0} \varphi(0) \mathrm{d} x=0
$$


and for any test function $\eta \in \mathcal{C}_{0}^{\infty}\left([0, T) \times \mathbb{R}^{3} ; \mathbb{R}^{3}\right)$ we have

$$
\begin{aligned}
& \int_{0}^{T} \int_{\mathbb{R}^{3}} J \cdot \partial_{t} \eta+\Lambda \otimes \Lambda: \nabla \eta+P(\rho) \operatorname{div} \eta-\rho \nabla V \cdot \eta \\
& +\hbar^{2} \nabla \sqrt{\rho} \otimes \nabla \sqrt{\rho}: \nabla \eta-\frac{\hbar^{2}}{4} \rho \Delta \operatorname{div} \eta \mathrm{d} x \mathrm{~d} t+\int_{\mathbb{R}^{3}} J_{0} \cdot \eta(0) \mathrm{d} x=0 ;
\end{aligned}
$$

- (generalized irrotationality condition) for almost every $t \in(0, T)$

$$
\nabla \wedge J=2 \nabla \sqrt{\rho} \wedge \Lambda
$$

holds in the sense of distributions.

We say that $(\rho, J)$ is a finite energy weak solution if and only if it is a weak solution and the energy $E(t)$ is finite a.e. in $[0, T)$.

The next existence result roughly speaking shows how to get a weak solution to the system (38) out of a strong solution to the SchrödingerPoisson system

$$
\left\{\begin{array}{l}
i \hbar \partial_{t} \psi+\frac{\hbar^{2}}{2} \Delta \psi=|\psi|^{p-1} \psi+V \psi \\
-\Delta V=|\psi|^{2}
\end{array}\right.
$$

The quadratic nonlinearities in (38) are originated by a term of the form $\operatorname{Re}(\nabla \bar{\psi} \otimes \nabla \psi)$ since formally

$$
\hbar^{2} \operatorname{Re}(\nabla \bar{\psi} \otimes \nabla \psi)=\hbar^{2} \nabla \sqrt{\rho} \otimes \nabla \sqrt{\rho}+\frac{J \otimes J}{\rho} .
$$

However this identity can be justified in the nodal region $\{\rho=0\}$ only by means of the polar factorization discussed in the previous section. Indeed, we stress that in the previous identity the right hand side is written in terms of $\rho$ and $J$ which do exist in the whole space $\mathbb{R}^{3}$, via the Madelung transform. However, the term $\frac{J \otimes J}{\rho}$ should be interpreted as $\Lambda \otimes \Lambda$, where $\Lambda$ is the Radon-Nykodim derivative of $J \mathrm{~d} x$ with respect to $\sqrt{\rho} \mathrm{d} x$. Unfortunately the Madelung transformations are unable to define $\Lambda$ on the whole $\mathbb{R}^{3}$, hence one needs to use the polar factorization discussed in the previous section to define $\Lambda$ in the whole $\mathbb{R}^{3}$.

Furthermore, the study of the existence of weak solutions of (38) is done with Cauchy data of the form $\left(\rho_{0}, J_{0}\right)=\left(\left|\psi_{0}\right|^{2}, \hbar \operatorname{Im}\left(\overline{\psi_{0}} \nabla \psi_{0}\right)\right)$, for some $\psi_{0} \in H^{1}\left(\mathbb{R}^{3} ; \mathbb{C}\right)$. These special initial data yield to consider the Cauchy problem for the QHD system only for solutions compatible with a wave mechanics point of view.

Proposition 19. Let $0<T<\infty$, let $\psi_{0} \in H^{1}\left(\mathbb{R}^{3}\right)$ and define the initial data for (38), $\left(\rho_{0}, J_{0}\right):=\left(\left|\psi_{0}\right|^{2}, \hbar \operatorname{Im}\left(\overline{\psi_{0}} \nabla \psi_{0}\right)\right)$. Then there exists a finite energy weak solution $(\rho, J)$ to the Cauchy problem (38) in the 
space-time slab $[0, T) \times \mathbb{R}^{3}$. Furthermore the energy $E(t)$ defined in $(7)$ is conserved for all times $t \in[0, T)$.

The idea behind the proof of this Proposition is the following. Let us consider the Schrödinger-Poisson system (41) with initial datum $\psi(0)=\psi_{0}$. It is well known that it is globally well-posed for initial data in $H^{1}\left(\mathbb{R}^{3}\right)$ (see $[8]$ ), and the solution satisfies $\psi \in \mathcal{C}^{0}\left(\mathbb{R} ; H^{1}\left(\mathbb{R}^{3}\right)\right.$ ). Then it makes sense to define for each time $t \in[0, T)$ the quantities $\rho(t):=|\psi(t)|^{2}, J(t):=\hbar \operatorname{Im}(\overline{\psi(t)} \nabla \psi(t))$ and we can see that $(\rho, J)$ is a finite energy weak solution of (38): indeed for the solution $\psi$ of (41) we have

$$
\partial_{t} \nabla \psi=\frac{i \hbar}{2} \Delta \nabla \psi-\frac{i}{\hbar} \nabla\left(\left(|\psi|^{p-1}+V\right) \psi\right)
$$

in the sense of distributions. Thus formally we get the following identities for $(\rho, J)$

$$
\begin{gathered}
\partial_{t} \rho=-\hbar \operatorname{Im}(\bar{\psi} \Delta \psi)=-\operatorname{div}(\hbar \operatorname{Im}(\bar{\psi} \nabla \psi)), \\
\partial_{t} J=\hbar \operatorname{Im}\left(\nabla \psi\left(-\frac{i \hbar}{2} \Delta \bar{\psi}+\frac{i}{\hbar}\left(|\psi|^{p-1}+V\right) \bar{\psi}\right)\right) \\
+\hbar \operatorname{Im}\left(\bar{\psi}\left(\frac{i \hbar}{2} \Delta \nabla \psi-\frac{i}{\hbar} \nabla\left(|\psi|^{p-1}+V\right) \psi-\frac{i}{\hbar}\left(|\psi|^{p-1}+V\right) \nabla \psi\right)\right) \\
=\frac{\hbar^{2}}{4} \nabla \Delta|\psi|^{2}-\hbar^{2} \operatorname{div}(\operatorname{Re}(\nabla \bar{\psi} \otimes \nabla \psi))-\frac{p-1}{p+1} \nabla\left(|\psi|^{p+1}\right)-|\psi|^{2} \nabla V .
\end{gathered}
$$

Thanks to the Lemma 15 we can write

$$
\hbar^{2} \operatorname{Re}(\nabla \bar{\psi} \otimes \nabla \psi)=\hbar^{2} \nabla \sqrt{\rho} \otimes \nabla \sqrt{\rho}+\Lambda \otimes \Lambda
$$

with $\sqrt{\rho}, \Lambda$ as in statement of the Lemma 15, and clearly they also satisfy

$$
J=\sqrt{\rho} \Lambda .
$$

Hence formally the following identity holds:

$$
\partial_{t} J+\operatorname{div}(\Lambda \otimes \Lambda)+\nabla P(\rho)+\rho \nabla V=\frac{\hbar^{2}}{4} \Delta \nabla \rho-\hbar^{2} \operatorname{div}(\nabla \sqrt{\rho} \otimes \nabla \sqrt{\rho}) .
$$

Of course these calculations are just formal, since $\psi$ doesn't have the necessary regularity to implement them.

Furthermore, it is well known that the energy

$$
E(t):=\int_{\mathbb{R}^{3}} \frac{\hbar^{2}}{2}|\nabla \psi|^{2}+\frac{2}{p+1}|\psi|^{p+1} \mathrm{~d} x+\frac{1}{2} \int_{\mathbb{R}_{x}^{3} \times \mathbb{R}_{y}^{3}}|\psi(t, y)|^{2} \frac{1}{|x-y|}|\psi(t, x)|^{2} \mathrm{~d} x \mathrm{~d} y
$$


is conserved for the Schrödinger-Poisson system (41). Thus, it only remains to note that by Lemma 15 the energy in (43) is equal that one in $(7)$.

Proof. Let us consider the Schrödinger-Poisson system (41). From standard theory about nonlinear Schrödinger equations it is well known that (41) is globally well-posed for initial data in the space of energy: $\psi(0)=\psi_{0} \in H^{1}\left(\mathbb{R}^{3}\right)$. Now, let us take a sequence of mollifiers $\left\{\chi_{\varepsilon}\right\}$ converging to the Dirac mass and define $\psi^{\varepsilon}:=\chi_{\varepsilon} \star \psi$, where $\psi \in \mathcal{C}\left(\mathbb{R} ; H^{1}\left(\mathbb{R}^{3}\right)\right)$ is the solution to (41). Then $\psi^{\varepsilon} \in \mathcal{C}^{\infty}\left(\mathbb{R}^{1+3}\right)$ and moreover

$$
i \hbar \partial_{t} \psi^{\varepsilon}+\frac{\hbar^{2}}{2} \Delta \psi^{\varepsilon}=\chi_{\varepsilon} \star\left(|\psi|^{p-1} \psi+V \psi\right),
$$

where $V$ is the classical Hartree potential. Therefore differentiating $\left|\psi^{\varepsilon}\right|^{2}$ with respect to time we get

$$
\begin{gathered}
\partial_{t}\left|\psi^{\varepsilon}\right|^{2}=2 \operatorname{Re}\left(\overline{\psi^{\varepsilon}} \partial_{t} \psi^{\varepsilon}\right)=2 \operatorname{Re}\left(\overline{\psi^{\varepsilon}}\left(\frac{i \hbar}{2} \Delta \psi^{\varepsilon}-\frac{i}{\hbar} \chi_{\varepsilon} \star\left(|\psi|^{p-1} \psi+V \psi\right)\right)\right) \\
=-\hbar \operatorname{div}\left(\operatorname{Im}\left(\overline{\psi^{\varepsilon}} \nabla \psi^{\varepsilon}\right)\right)+\frac{2}{\hbar} \operatorname{Im}\left(\overline{\psi^{\varepsilon}} \chi_{\varepsilon} \star\left(|\psi|^{p-1} \psi+V \psi\right)\right) .
\end{gathered}
$$

If we differentiate with respect to time $\hbar \operatorname{Im}\left(\overline{\psi^{\varepsilon}} \nabla \psi^{\varepsilon}\right)$ we get

$$
\begin{gathered}
\partial_{t}\left(\hbar \operatorname{Im}\left(\overline{\psi^{\varepsilon}} \nabla \psi^{\varepsilon}\right)\right)=\hbar \operatorname{Im}\left[\left(-\frac{i \hbar}{2} \Delta \overline{\psi^{\varepsilon}}+\frac{i}{\hbar} \chi_{\varepsilon} \star\left(|\psi|^{p-1} \bar{\psi}\right)+\frac{i}{\hbar} \chi_{\varepsilon} \star(V \psi)\right)\right] \\
+\hbar \operatorname{Im}\left[\overline{\psi^{\varepsilon}}\left(\frac{i \hbar}{2} \delta \nabla \psi^{\varepsilon}-\frac{i}{\hbar} \chi_{\varepsilon} \star \nabla\left(|\psi|^{p-1} \psi\right)-\frac{i}{\hbar} \chi_{\varepsilon} \star \nabla(V \psi)\right)\right] \\
=\frac{\hbar^{2}}{2} \operatorname{Re}\left(-\nabla \psi^{\varepsilon} \Delta \overline{\psi^{\varepsilon}}+\overline{\psi^{\varepsilon}} \Delta \nabla \psi^{\varepsilon}\right)+\operatorname{Re}\left(\chi_{\varepsilon} \star\left(|\psi|^{p-1} \bar{\psi}\right) \nabla \psi^{\varepsilon}-\overline{\psi^{\varepsilon}} \chi_{\varepsilon} \star \nabla\left(|\psi|^{p-1} \psi\right)\right) \\
+\operatorname{Re}\left(\chi \star(V \bar{\psi}) \nabla \psi^{\varepsilon}-\chi_{\varepsilon} \star \nabla(V \psi) \overline{\psi^{\varepsilon}}\right)=: A+B+C
\end{gathered}
$$

Let us discuss these three terms separately. For the first term $A$ it is immediate that

$$
\frac{\hbar^{2}}{2} \operatorname{Re}\left(-\nabla \psi^{\varepsilon} \Delta \overline{\psi^{\varepsilon}}+\overline{\psi^{\varepsilon}} \Delta \nabla \psi^{\varepsilon}\right)=\hbar^{2} \operatorname{div}\left(\operatorname{Re}\left(\nabla \psi^{\varepsilon} \otimes \nabla \overline{\psi^{\varepsilon}}\right)\right)+\frac{\hbar^{2}}{4} \Delta \nabla\left|\psi^{\varepsilon}\right|^{2} .
$$

Let us discuss the second and the third terms, $B$ and $C$. We can recast $B$ in the following way

$$
\begin{gathered}
\chi_{\varepsilon} \star\left(|\psi|^{p-1} \bar{\psi}\right) \nabla \psi^{\varepsilon}-\overline{\psi^{\varepsilon}} \chi_{\varepsilon} \star \nabla\left(|\psi|^{p-1} \psi\right) \\
=\left|\psi^{\varepsilon}\right|^{p-1} \overline{\psi^{\varepsilon}} \nabla \psi^{\varepsilon}-\overline{\psi^{\varepsilon}} \chi_{\varepsilon} \star\left(\psi \nabla|\psi|^{p-1}+|\psi|^{p-1} \nabla \psi\right)+R_{1}^{\varepsilon} \\
=-\overline{\psi^{\varepsilon}} \chi_{\varepsilon} \star\left(\psi \nabla|\psi|^{p-1}\right)+R_{1}^{\varepsilon}+R_{2}^{\varepsilon}=-\left|\psi^{\varepsilon}\right|^{2} \nabla\left|\psi^{\varepsilon}\right|^{p-1}+R_{1}^{\varepsilon}+R_{2}^{\varepsilon}+R_{3}^{\varepsilon},
\end{gathered}
$$


where

$$
\begin{gathered}
R_{1}^{\varepsilon}:=\left(\chi_{\varepsilon} \star\left(|\psi|^{p-1} \bar{\psi}\right)-\left|\psi^{\varepsilon}\right|^{p-1} \overline{\psi^{\varepsilon}}\right) \nabla \psi^{\varepsilon} \\
R_{2}^{\varepsilon}:=\overline{\psi^{\varepsilon}}\left(\left|\psi^{\varepsilon}\right|^{p-1} \nabla \psi^{\varepsilon}-\chi_{\varepsilon} \star\left(|\psi|^{p-1} \nabla \psi\right)\right) \\
R_{3}^{\varepsilon}:=\overline{\psi^{\varepsilon}}\left(\psi^{\varepsilon} \nabla\left|\psi^{\varepsilon}\right|^{p-1}-\chi_{\varepsilon} \star\left(\psi \nabla|\psi|^{p-1}\right)\right) .
\end{gathered}
$$

Now it only remains to analyze how the remainder terms $R_{j}^{\varepsilon}$ go to zero. We will prove it in the next Lemma. Furthermore, the following identity holds

$$
\left|\psi^{\varepsilon}\right|^{2} \nabla\left|\psi^{\varepsilon}\right|^{p-1}=\frac{p-1}{p+1} \nabla\left|\psi^{\varepsilon}\right|^{p+1} .
$$

For the third term $C$ after similar computations we get

$$
C=\left|\psi^{\varepsilon}\right| \nabla\left(\chi_{\varepsilon} \star V\right)+R_{4}^{\varepsilon},
$$

where the remainder term $R_{4}^{\varepsilon}$ will be analyzed in the next Lemma. Hence we can conclude that $\hbar \operatorname{Im}\left(\overline{\psi^{\varepsilon}} \nabla \psi^{\varepsilon}\right)$ satisfies

$$
\begin{aligned}
\partial_{t}\left(\hbar \operatorname{Im}\left(\overline{\psi^{\varepsilon}} \nabla \psi^{\varepsilon}\right)\right)=-\hbar^{2} \operatorname{div} & \left(\operatorname{Re}\left(\nabla \psi^{\varepsilon} \otimes \nabla \overline{\psi^{\varepsilon}}\right)\right)+\frac{\hbar^{2}}{4} \Delta \nabla\left|\psi^{\varepsilon}\right|^{2} \\
+ & \frac{p-1}{p+1} \nabla\left|\psi^{\varepsilon}\right|^{p+1}+\left|\psi^{\varepsilon}\right|^{2} \nabla\left(\chi_{\varepsilon} \star V\right)+R^{\varepsilon},
\end{aligned}
$$

where $R^{\varepsilon}:=R_{1}^{\varepsilon}+R_{2}^{\varepsilon}+R_{3}^{\varepsilon}+R_{4}^{\varepsilon}$. Now, as $\varepsilon \rightarrow 0$, we get

$$
\begin{aligned}
\partial_{t}(\hbar \operatorname{Im}(\bar{\psi} \nabla \psi))=-\hbar^{2} \operatorname{div}(\operatorname{Re}(\nabla \psi \otimes \nabla \bar{\psi})) & +\frac{\hbar^{2}}{4} \Delta \nabla|\psi|^{2} \\
& +\frac{p-1}{p+1} \nabla|\psi|^{p+1}+|\psi|^{2} \nabla V .
\end{aligned}
$$

This identity is equivalent to (42) since by the Lemma 15 we have

$$
\hbar^{2} \operatorname{Re}(\nabla \psi \otimes \nabla \psi)=\hbar^{2} \nabla \sqrt{\rho} \otimes \nabla \sqrt{\rho}+\Lambda \otimes \Lambda,
$$

where $\Lambda$ is defined as in the Lemma 15 and $\sqrt{\rho} \Lambda=J$, where $J:=$ $\hbar \operatorname{Im}(\bar{\psi} \nabla \psi)$.

Finally, let us note that, by the definition of $J$, we have

$$
\nabla \wedge J=\hbar \operatorname{Im}(\nabla \bar{\psi} \wedge \nabla \psi)
$$

then by the Corollary 16, we get $\nabla \wedge J=2 \nabla \sqrt{\rho} \wedge \Lambda$.

Lemma 20. Let $0<T<\infty$, then

$$
\left\|R_{j}^{\varepsilon}\right\|_{L_{t, x}^{1}\left([0, T] \times \mathbb{R}^{3}\right)} \rightarrow 0, \quad \text { as } \varepsilon \rightarrow 0,
$$

$j=1,2,3,4$. 
Proof. It is a direct consequence of Strichartz estimates for the solution of the Schrödinger-Poisson system (41)

$$
\|\psi\|_{L_{t}^{q} W^{1, r}\left([0, T] \times \mathbb{R}^{3}\right)} \leq C\left(E_{0},\left\|\psi_{0}\right\|_{L^{2}\left(\mathbb{R}^{3}\right)}, T\right),
$$

where $(q, r)$ is a pair of admissible exponents and $E_{0}$ is the initial energy for (41). The first error can be controlled in the following way

$$
\begin{aligned}
& \left\|R_{1}^{\varepsilon}\right\|_{L_{t, x}^{1}\left([0, T] \times \mathbb{R}^{3}\right)} \\
& \leq\left\|\chi_{\varepsilon} \star\left(|\psi|^{p-1} \bar{\psi}\right)-\left|\psi^{\varepsilon}\right|^{p-1} \overline{\psi^{\varepsilon}}\right\|_{L_{t}^{\frac{4(p+1)}{p+7}} L_{x}^{\frac{p+1}{p}}\left([0, T] \times \mathbb{R}^{3}\right)}\left\|\nabla \psi^{\varepsilon}\right\|_{L_{t}^{\frac{4(p+1)}{3(p-1)}} L_{x}^{p+1}\left([0, T] \times \mathbb{R}^{3}\right)} \\
& \leq T^{\frac{p+7}{4(p+1)}}\left(\left\|\chi_{\varepsilon} \star\left(|\psi|^{p-1} \bar{\psi}\right)-|\psi|^{p-1} \bar{\psi}\right\|_{L_{t}^{\infty} L_{x}^{p+1}}\right. \\
& \left.+\left\||\psi|^{p-1} \bar{\psi}-\left|\psi^{\varepsilon}\right|^{p-1} \overline{\psi^{\varepsilon}}\right\|_{L_{t}^{\infty} L_{x}^{p+1}}\right)\left\|\nabla \psi^{\varepsilon}\right\|_{L_{t}^{\frac{3(p-1)}{4(p+1)}} L_{x}^{p+1}} .
\end{aligned}
$$

The remaining error terms can be computed in the same way. We remark that by using the Strichartz estimates it folllows $\psi \nabla|\psi|^{p-1}$ lies in $L_{t}^{\frac{4(p+1)}{3(p-1)}} L_{x}^{p+1}\left([0, T] \times \mathbb{R}^{3}\right)$.

\section{THE FRACTIONAL STEP: DEFINITIONS AND CONSISTENCY}

In this section we make use of the results of the previous sections to construct a sequence of approximate solutions of the QHD system

$$
\left\{\begin{array}{l}
\partial_{t} \rho+\operatorname{div} J=0 \\
\partial_{J}+\operatorname{div}\left(\frac{J \otimes J}{\rho}\right)+\nabla P(\rho)+\rho \nabla V+J=\frac{\hbar^{2}}{2} \rho \nabla\left(\frac{\Delta \sqrt{\rho}}{\sqrt{\rho}}\right) \\
-\Delta V=\rho
\end{array}\right.
$$

with Cauchy data

$$
(\rho(0), J(0))=\left(\rho_{0}, J_{0}\right) .
$$

Definition 21. Let $0<T<\infty$. We say $\left\{\left(\rho^{\tau}, J^{\tau}\right)\right\}$ is a sequence of approximate solutions to the system $(1)$ in $[0, T) \times \mathbb{R}^{3}$, with initial data $\left(\rho_{0}, J_{0}\right) \in L_{\text {loc }}^{1}\left(\mathbb{R}^{3}\right)$, if there exist locally integrable functions $\sqrt{\rho^{\tau}}, \Lambda^{\tau}$, such that $\sqrt{\rho^{\tau}} \in L_{l o c}^{2}\left([0, T) ; H_{l o c}^{1}\left(\mathbb{R}^{3}\right)\right), \Lambda^{\tau} \in L_{l o c}^{2}\left([0, T) ; L_{l o c}^{2}\left(\mathbb{R}^{3}\right)\right)$ and if we define $\rho^{\tau}:=\left(\sqrt{\rho^{\tau}}\right)^{2}, J^{\tau}:=\sqrt{\rho^{\tau}} \Lambda^{\tau}$, then

- for any test function $\eta \in \mathcal{C}_{0}^{\infty}\left([0, T) \times \mathbb{R}^{3}\right)$ one has

$$
\int_{0}^{T} \int_{\mathbb{R}^{3}} \rho^{\tau} \partial_{t} \eta+J^{\tau} \cdot \nabla \eta \mathrm{d} x \mathrm{~d} t+\int_{\mathbb{R}^{3}} \rho_{0} \eta(0) \mathrm{d} x=o(1)
$$


as $\tau \rightarrow 0$,

- for any test function $\zeta \in \mathcal{C}_{0}^{\infty}\left([0, T) \times \mathbb{R}^{3} ; \mathbb{R}^{3}\right)$ we have

$$
\begin{aligned}
& \int_{0}^{T} \int_{\mathbb{R}^{3}} J^{\tau} \cdot \partial_{t} \zeta+\Lambda^{\tau} \otimes \Lambda^{\tau}: \nabla \zeta+P\left(\rho^{\tau}\right) \operatorname{div} \zeta-\rho^{\tau} \nabla V^{\tau} \cdot \zeta-J^{\tau} \cdot \zeta \\
& +\hbar^{2} \nabla \sqrt{\rho^{\tau}} \otimes \nabla \sqrt{\rho^{\tau}}: \nabla \zeta-\frac{\hbar^{2}}{4} \rho^{\tau} \Delta \operatorname{div} \zeta \mathrm{d} x \mathrm{~d} t+\int_{\mathbb{R}^{3}} J_{0} \cdot \zeta(0) \mathrm{d} x=o(1) \\
& \text { as } \tau \rightarrow 0 ;
\end{aligned}
$$

- (generalized irrotationality condition) for almost every $t \in(0, T)$ we have

$$
\nabla \wedge J^{\tau}=2 \nabla \sqrt{\rho^{\tau}} \wedge \Lambda^{\tau}
$$

Our fractional step method is based on the following simple idea. We split the evolution of our problem into two separate steps. Let us fix a (small) parameter $\tau>0$ then in the former step we solve a noncollisional QHD problem, while in the latter one we solve the collisional problem without QHD, and at this point we can start again with the non-collisional QHD problem, the main difficulty being the updating of the initial data at each time step. Indeed, as remarked in the previous section we are able to solve the non-collisional QHD only in the case of Cauchy data compatible with the Schrödinger picture. This restriction impose to reconstruct a wave function at each time step.

Now we explain how to set up the fractional step procedure which generates the approximate solutions. We first remark that, as in the previous section, this method requires special type of initial data $\left(\rho_{0}, J_{0}\right)$, namely we assume there exists $\psi_{0} \in H^{1}\left(\mathbb{R}^{3}\right)$, such that the hydrodynamic initial data are given via the Madelung transforms

$$
\rho_{0}=\left|\psi_{0}\right|^{2}, \quad J_{0}=\hbar \operatorname{Im}\left(\overline{\psi_{0}} \nabla \psi_{0}\right) .
$$

This assumption is physically relevant since it implies the compatibility of our solutions to the QHD problem with the wave mechanics approach. The iteration procedure can be defined in this following way. First of all, we take $\tau>0$, which will be the time mesh unit; therefore we define the approximate solutions in each time interval $[k \tau,(k+1) \tau)$, for any integer $k \geq 0$.

At the first step, $k=0$, we solve the Cauchy problem for the SchrödingerPoisson system

$$
\left\{\begin{array}{l}
i \hbar \partial_{t} \psi^{\tau}+\frac{\hbar^{2}}{2} \Delta \psi^{\tau}=\left|\psi^{\tau}\right|^{p-1} \psi^{\tau}+V^{\tau} \psi^{\tau} \\
-\Delta V^{\tau}=\left|\psi^{\tau}\right|^{2} \\
\psi^{\tau}(0)=\psi_{0}
\end{array}\right.
$$


by looking for the restriction in $[0, \tau)$ of the unique strong solution $\psi \in$ $\mathcal{C}\left(\mathbb{R} ; H^{1}\left(\mathbb{R}^{3}\right)\right.$ ) (see $\left.[8]\right)$. Let us define $\rho^{\tau}:=\left|\psi^{\tau}\right|^{2}, J^{\tau}:=\hbar \operatorname{Im}\left(\overline{\psi^{\tau}} \nabla \psi^{\tau}\right)$. Then, as shown in the previous section, $\left(\rho^{\tau}, J^{\tau}\right)$ is a weak solution to the non-collisional QHD system. Let us assume that we know $\psi^{\tau}$ in the space-time slab $[(k-1) \tau, k \tau) \times \mathbb{R}^{3}$, we want to set up a recursive method, hence we have to show how to define $\psi^{\tau}, \rho^{\tau}, J^{\tau}$ in the strip $[k \tau,(k+1) \tau)$.

In order to take into account the presence of the collisional term $f=J$ we update $\psi$ in $t=k \tau$, namely we define $\psi^{\tau}(k \tau+)$. The construction of $\psi^{\tau}(k \tau+)$ will be done by means of the polar decomposition described in the Section 3.

Let us apply the Lemma 17, with $\psi=\psi^{\tau}(k \tau-), \varepsilon=\tau 2^{-k}\left\|\psi_{0}\right\|_{H^{1}\left(\mathbb{R}^{3}\right)}$, then we can define

$$
\psi^{\tau}(k \tau+)=\tilde{\psi}
$$

by using the wave function $\tilde{\psi}$ defined in the Lemma 17 . Therefore we have

$$
\begin{aligned}
\rho^{\tau}(k \tau+) & =\rho^{\tau}(k \tau-) \\
\Lambda^{\tau}(k \tau+) & =(1-\tau) \Lambda^{\tau}(k \tau-)+R_{k},
\end{aligned}
$$

where $\left\|R_{k}\right\|_{L^{2}\left(\mathbb{R}^{3}\right)} \leq \tau 2^{-k}\left\|\psi_{0}\right\|_{H^{1}\left(\mathbb{R}^{3}\right)}$ and

$$
\nabla \psi^{\tau}(k \tau+)=\nabla \psi^{\tau}(k \tau-)-i \frac{\tau}{\hbar} \phi^{*} \Lambda^{\tau}(k \tau-)+r_{k, \tau}
$$

with $\left\|\phi^{*}\right\|_{L^{\infty}} \leq 1$ and

$$
\left\|r_{k, \tau}\right\|_{L^{2}} \leq C\left(\tau\left\|\nabla \psi^{\tau}(k \tau-)\right\|+\tau 2^{-k}\left\|\psi_{0}\right\|_{H^{1}\left(\mathbb{R}^{3}\right)}\right) \lesssim \tau E_{0}^{\frac{1}{2}}
$$

We then solve the Schrödinger-Poisson system with initial data $\psi(0)=$ $\psi^{\tau}(k \tau+)$. We define $\psi^{\tau}$ in the time strip $[k \tau,(k+1) \tau)$ as the restriction of the Schrödinger-Poisson solution just found in $[0, \tau)$, furthermore, we define $\rho^{\tau}:=\left|\psi^{\tau}\right|^{2}, J^{\tau}:=\hbar \operatorname{Im}\left(\overline{\psi^{\tau}} \nabla \psi^{\tau}\right)$ as the solution of the noncollisional QHD (38).

With this procedure we can go on every time strip and then construct an approximate solution $\left(\rho^{\tau}, J^{\tau}, V^{\tau}\right)$ of the QHD system.

Remark 22. To cover the general case where $f(\sqrt{\rho}, J, \nabla \sqrt{\rho})=\alpha J+$ $\rho \nabla g(t, x, \sqrt{\rho}, \Lambda, \nabla \sqrt{\rho}), \alpha \geq 0, g$ satisfies the conditions in the Remark 5 and one has a non-zero doping profile, we solve the following Cauchy 
problem

$$
\left\{\begin{array}{l}
i \hbar \partial_{t} \psi+\frac{\hbar^{2}}{2} \Delta \psi=|\psi|^{p-1} \psi+V \psi+g \psi \\
-\Delta V=|\psi|^{2}-C(x) \\
\psi(0)=\psi_{0}
\end{array}\right.
$$

The global well-posedness follows from the result in [8].

Theorem 23 (Consistency of the approximate solutions). Let us consider the sequence of approximate solutions $\left\{\left(\rho^{\tau}, J^{\tau}\right)\right\}_{\tau>0}$ constructed via the fractional step method, and assume there exists $0<T<\infty$, $\sqrt{\rho} \in L_{l o c}^{2}\left([0, T) ; H_{l o c}^{1}\left(\mathbb{R}^{3}\right)\right)$ and $\Lambda \in L_{l o c}^{2}\left([0, T) ; L_{l o c}^{2}\left(\mathbb{R}^{3}\right)\right)$, such that

$$
\begin{array}{cc}
\sqrt{\rho^{\tau}} \rightarrow \sqrt{\rho} & \text { in } L^{2}\left([0, T) ; H_{l o c}^{1}\left(\mathbb{R}^{3}\right)\right) \\
\Lambda^{\tau} & \rightarrow \Lambda \quad \text { in } L^{2}\left([0, T) ; L_{l o c}^{2}\left(\mathbb{R}^{3}\right)\right) .
\end{array}
$$

Then the limit function $(\rho, J)$, where as before $J=\sqrt{\rho} \Lambda$, is a weak solution of the QHD system, with Cauchy data $\left(\rho_{0}, J_{0}\right)$.

Proof. Let us plug the approximate solutions $\left(\rho^{\tau}, J^{\tau}\right)$ in the weak formulation and let $\zeta \in \mathcal{C}_{0}^{\infty}\left([0, T) \times \mathbb{R}^{3}\right)$, then we have

$$
\begin{gathered}
\int_{0}^{\infty} \int_{\mathbb{R}^{3}} J^{\tau} \cdot \partial_{t} \zeta+\Lambda^{\tau} \otimes \Lambda^{\tau}: \nabla \zeta+P\left(\rho^{\tau}\right) \operatorname{div} \zeta-\rho^{\tau} \nabla V^{\tau} \cdot \zeta-J^{\tau} \cdot \zeta \\
+\hbar^{2} \nabla \sqrt{\rho^{\tau}} \otimes \nabla \sqrt{\rho^{\tau}}: \nabla \zeta-\frac{\hbar^{2}}{4} \rho^{\tau} \Delta \operatorname{div} \zeta \mathrm{d} x \mathrm{~d} t+\int_{\mathbb{R}^{3}} J_{0} \cdot \zeta(0) \mathrm{d} x \\
=\sum_{k=0}^{\infty} \int_{k \tau}^{(k+1) \tau} \int_{\mathbb{R}^{3}} J^{\tau} \cdot \partial_{t} \zeta+\Lambda^{\tau} \otimes \Lambda^{\tau}: \nabla \zeta+P\left(\rho^{\tau}\right) \operatorname{div} \zeta-\rho^{\tau} \nabla V^{\tau} \cdot \zeta-J^{\tau} \cdot \zeta \\
+\hbar^{2} \nabla \sqrt{\rho^{\tau}} \otimes \nabla \sqrt{\rho^{\tau}}: \nabla \zeta-\frac{\hbar^{2}}{4} \rho^{\tau} \Delta \operatorname{div} \zeta \mathrm{d} x \mathrm{~d} t+\int_{\mathbb{R}^{3}} J_{0} \cdot \zeta(0) \mathrm{d} x \\
=\sum_{k=0}^{\infty}\left[\int_{k \tau}^{(k+1) \tau} \int_{\mathbb{R}^{3}}-J^{\tau} \cdot \zeta \mathrm{d} x \mathrm{~d} t+\int_{\mathbb{R}^{3}} J^{\tau}((k+1) \tau-) \cdot \zeta((k+1) \tau)\right. \\
\left.-J^{\tau}(k \tau) \cdot \zeta(k \tau) \mathrm{d} x\right]+\int_{\mathbb{R}^{3}} J_{0} \cdot \zeta(0) \mathrm{d} x
\end{gathered}
$$




$$
\begin{gathered}
=\sum_{k=0}^{\infty} \int_{k \tau}^{(k+1) \tau} \int_{\mathbb{R}^{3}}-J^{\tau} \cdot \zeta \mathrm{d} x \mathrm{~d} t+\sum_{k=1}^{\infty} \int_{\mathbb{R}^{3}}\left(J^{\tau}(k \tau-)-J^{\tau}(k \tau)\right) \cdot \zeta(k \tau) \mathrm{d} x \\
=\sum_{k=0}^{\infty} \int_{k \tau}^{(k+1) \tau} \int_{\mathbb{R}^{3}}-J^{\tau} \cdot \zeta \mathrm{d} x \mathrm{~d} t+\sum_{k=1}^{\infty} \tau \int_{\mathbb{R}^{3}} J^{\tau}(k \tau-) \cdot \zeta(k \tau) \mathrm{d} x \\
+\sum_{k=1}^{\infty} \int_{\mathbb{R}^{3}}(1-\tau) \sqrt{\rho^{\tau}(k \tau)} R_{k} \cdot \zeta(k \tau) \mathrm{d} x \\
=\sum_{k=0}^{\infty} \int_{k \tau}^{(k+1) \tau} \int_{\mathbb{R}^{3}} J^{\tau}((k+1) \tau-) \cdot \zeta((k+1) \tau)-J^{\tau}(t) \cdot \zeta(t) \mathrm{d} x \mathrm{~d} t \\
+(1-\tau) \sum_{k=0}^{\infty} \int_{\mathbb{R}^{3}} \sqrt{\rho^{\tau}}(k \tau) R_{k} \cdot \zeta(k \tau) \mathrm{d} x \\
=o(1)+O(\tau)
\end{gathered}
$$

as $\tau \rightarrow 0$.

\section{A PRIORI ESTIMATES AND CONVERGENCE}

In this section we obtain various a priori estimates necessary to show the compactness of the sequence of approximate solutions $\left(\rho^{\tau}, J^{\tau}\right)$ in some appropriate function spaces. As we stated in the Theorem 23, we wish to prove the strong convergence of $\left\{\sqrt{\rho^{\tau}}\right\}$ in $L_{l o c}^{2}\left([0, T) ; H_{l o c}^{1}\left(\mathbb{R}^{3}\right)\right)$ and that one of $\left\{\Lambda^{\tau}\right\}$ in $L_{l o c}^{2}\left([0, T) ; L_{l o c}^{2}\left(\mathbb{R}^{3}\right)\right)$. To achieve this goal we use a compactness result in the class of the Aubin-Lions's type lemma, due to Rakotoson-Temam [44] (see the Section 2). The plan of this section is the following, first of all we get a discrete version of the (dissipative) energy inequality for the system (1), later we use the Strichartz estimates for $\nabla \psi^{\tau}$ by means of the formula (61) below. Consequently via the Strichartz estimates and by using the local smoothing results of Theorems 11, 12, we deduce some further regularity properties of the sequence $\left\{\nabla \psi^{\tau}\right\}$. This fact will be stated in the next Proposition 33. In this way it is possible to get the regularity properties of the sequence $\left\{\nabla \psi^{\tau}\right\}$ which are needed to apply the Theorem 13 and hence to get the convergence for $\left\{\sqrt{\rho^{\tau}}\right.$ and $\left\{\Lambda^{\tau}\right\}$.

Let us begin with the energy inequality. First of all note that if we have a sufficiently regular solution of the QHD system, one has

$$
E(t)=-\int_{0}^{t} \int_{\mathbb{R}^{3}}|\Lambda|^{2} \mathrm{~d} x \mathrm{~d} t^{\prime}+E_{0},
$$


where the energy is defined as in (7). Now we would like to find a discrete version of the energy dissipation for the approximate solutions.

Proposition 24 (Discrete energy inequality). Let $\left(\rho^{\tau}, J^{\tau}\right)$ be an approximate solution of the QHD system, with $0<\tau<1$. Then, for $t \in[N \tau,(N+1) \tau)$ we have

$$
E^{\tau}(t) \leq-\frac{\tau}{2} \sum_{k=1}^{N}\|\Lambda(k \tau-)\|_{L^{2}\left(\mathbb{R}^{3}\right)}+(1+\tau) E_{0} .
$$

Proof. For all $k \geq 1$, we have

$$
\begin{aligned}
E^{\tau}(k \tau+)-E^{\tau}(k \tau-)= & \int \frac{1}{2}\left|\Lambda^{\tau}(k \tau+)\right|^{2}-\frac{1}{2}\left|\Lambda^{\tau}(k \tau-)\right|^{2} \mathrm{~d} x \\
= & \frac{1}{2} \int\left(-2 \tau+\tau^{2}\right)\left|\Lambda^{\tau}(k \tau-)\right|^{2} \\
& \quad+2(1-\tau) \Lambda^{\tau}(k \tau-) \cdot R_{k}+\left|R_{k}\right|^{2} \mathrm{~d} x \\
\leq & \frac{1}{2} \int\left(-2 \tau+\tau^{2}\right)\left|\Lambda^{\tau}(k \tau-)\right|^{2} \mathrm{~d} x+(1-\tau) \alpha\left|\Lambda^{\tau}(k \tau-)\right|^{2} \\
& \quad+\frac{1-\tau}{\alpha}\left|R_{k}\right|^{2}+\left|R_{k}\right|^{2} \mathrm{~d} x \\
= & \frac{1}{2} \int\left(-2 \tau+\tau^{2}+\alpha-\alpha \tau\right)\left|\Lambda^{\tau}(k \tau-)\right|^{2} \\
& \quad+\left(\frac{1-\tau+\alpha}{\alpha}\right)\left|R_{k}\right|^{2} \mathrm{~d} x .
\end{aligned}
$$

Here $R_{k}$ denotes the error term as in espression (53). If we choose $\alpha=\tau$, it follows

$$
\begin{aligned}
E^{\tau}(k \tau+)-E^{\tau}(k \tau-) \leq- & \frac{\tau}{2}\left\|\Lambda^{\tau}(k \tau-)\right\|_{L^{2}}^{2}+\frac{1}{2 \tau}\left\|R_{k}\right\|_{L^{2}}^{2} \\
& \leq-\frac{\tau}{2}\left\|\Lambda^{\tau}(k \tau-)\right\|_{L^{2}}^{2}+\tau 2^{-k-1}\left\|\psi_{0}\right\|_{H^{1}} .
\end{aligned}
$$

The inequality (59) follows by summing up all the terms in (60) and by the energy conservation in each time strip $[k \tau,(k+1) \tau)$.

Unfortunately the energy estimates are not sufficient to get enough compactness to show the convergence of the sequence of the approximate solutions. Indeed from the discrete energy inequality, we get only the weak convergence of $\nabla \psi^{\tau}$ in $L^{\infty}\left([0, \infty) ; H^{1}\left(\mathbb{R}^{3}\right)\right)$, and therefore the quadratic terms in (49) could exhibit some concentrations phenomena in the limit. 
More precisely, from energy inequality we get the sequence $\left\{\psi^{\tau}\right\}$ is uniformly bounded in $L^{\infty}\left([0, \infty) ; H^{1}\left(\mathbb{R}^{3}\right)\right)$, hence there exists $\psi \in L^{\infty}\left([0, \infty) ; H^{1}\left(\mathbb{R}^{3}\right)\right)$, such that

$$
\psi^{\tau} \rightarrow \psi \quad \text { in } L^{\infty}\left([0, \infty) ; H^{1}\left(\mathbb{R}^{3}\right)\right) .
$$

Therefore we get

$$
\begin{array}{cc}
\sqrt{\rho^{\tau}} \rightarrow \sqrt{\rho} & \text { in } L^{\infty}\left([0, \infty) ; H^{1}\left(\mathbb{R}^{3}\right)\right) \\
\Lambda^{\tau} \rightarrow \Lambda \quad \text { in } L^{\infty}\left([0, \infty) ; L^{2}\left(\mathbb{R}^{3}\right)\right) & \\
J^{\tau} \rightarrow \sqrt{\rho} \Lambda \quad \text { in } L^{\infty}\left([0, \infty) ; L_{l o c}^{1}\left(\mathbb{R}^{3}\right)\right) .
\end{array}
$$

The need to pass into the limit the quadratic expressions leads us to look for a priori estimates in stronger norms. The relationships with the Schrödinger equation brings naturally into this search the Strichartz-type estimates. The following results are concerned with these estimates. However they are not an immediate consequence of the Strichartz estimates for the Schrödinger equation since we have to take into account the effects of the updating procedure which we implement at each time step.

The following Remark 27 will clarify in detail why we stated the Lemma 17 in place of using the exact factorization property provided by the Lemma 15.

Lemma 25. Let $\psi^{\tau}$ be the wave function defined by the fractional step method (see Section 5), and let $t \in[N \tau,(N+1) \tau)$. Then we have

$$
\begin{aligned}
\nabla \psi^{\tau}(t)=U(t) \nabla \psi_{0} & -i \frac{\tau}{\hbar} \sum_{k=1}^{N} U(t-k \tau)\left(\phi_{k}^{\tau} \Lambda^{\tau}(k \tau-)\right) \\
& -i \int_{0}^{t} U(t-s) F(s) \mathrm{d} s+\sum_{k=1}^{N} U(t-k \tau) r_{k}^{\tau}
\end{aligned}
$$

where as before $U(t)$ is the free Schrödinger group,

$$
\left\|\phi_{k}^{\tau}\right\|_{L^{\infty}\left(\mathbb{R}^{3}\right)} \leq 1, \quad\left\|r_{k}^{\tau}\right\|_{L^{2}\left(\mathbb{R}^{3}\right)} \leq \tau\left\|\psi_{0}\right\|_{H^{1}\left(\mathbb{R}^{3}\right)}
$$

and $F=\nabla\left(\left|\psi^{\tau}\right|^{p-1} \psi^{\tau}+V^{\tau} \psi^{\tau}\right)$.

Remark 26. Clearly, with a general collision term $f(\sqrt{\rho}, J, \nabla \sqrt{\rho})$ (see the Remark 5) and a non-zero doping profile $C(x)$ one has $F$ defined by $F=\nabla\left(\left|\psi^{\tau}\right|^{p-1} \psi^{\tau}+V^{\tau} \psi^{\tau}+g^{\tau} \psi^{\tau}\right)$, where now $V^{\tau}$ solves

$$
-\Delta V^{\tau}=\left|\psi^{\tau}\right|^{2}-C(x)
$$

and $g^{\tau}=g\left(t, x, \sqrt{\rho^{\tau}}, \Lambda^{\tau}, \nabla \sqrt{\rho^{\tau}}\right)$. 
Remark 27. Now we can see why the updating step in (51) has been defined through the Lemma 17. Indeed, one could possibly try to use the exact unitary factor as in the Lemma 15, namely $\psi^{\tau}(k \tau+):=\phi^{(1-\tau)} \sqrt{\rho}$, where $\phi, \sqrt{\rho}$ are respectively the unitary factor and the amplitude of $\psi^{\tau}(k \tau-)$ (note that $\phi$ is uniquely determined $\sqrt{\rho} \mathrm{d} x$, and $|\phi|=1, \sqrt{\rho} \mathrm{d} x$ a.e.) The meaning of this definition is clear if we approximate $\psi^{\tau}(k \tau-)$ (as in the proof of the Lemma 17) with smooth $\psi_{n}=e^{i \theta_{n}} \sqrt{\rho_{n}}$ and then we update $\psi_{n}$ with $\tilde{\psi}_{n}:=e^{i(1-\tau) \theta_{n}} \sqrt{\rho_{n}}$. Hence $\psi(k \tau+):=\lim _{n \rightarrow \infty} \tilde{\psi}_{n}$ in $H^{1}\left(\mathbb{R}^{3}\right)$. Moreover in this case we get the exact updating formulas

$$
\begin{aligned}
\left|\psi^{\tau}(k \tau+)\right|^{2} & =\left|\psi^{\tau}(k \tau)\right|^{2} \\
\hbar \operatorname{Im}\left(\overline{\psi^{\tau}} \nabla \psi^{\tau}\right)(k \tau+) & =(1-\tau) \hbar \operatorname{Im}\left(\overline{\psi^{\tau}} \nabla \psi^{\tau}\right)(k \tau-),
\end{aligned}
$$

without the small error $r_{\varepsilon}$ in the expression for the density current, however instead of the formula (61) we would find

$$
\begin{aligned}
\nabla \psi^{\tau}(t)= & U(t-N \tau) \sigma_{N \tau}^{\tau} U(\tau) \ldots \sigma_{\tau}^{\tau} U(\tau) \nabla \psi_{0} \\
& -i \frac{\tau}{\hbar} U(t-N \tau) \sigma_{N \tau}^{\tau} \Lambda^{\tau}(N \tau-)+\ldots-i \frac{\tau}{\hbar} U(t-N \tau) \ldots U(\tau) \phi_{\tau}^{\tau} \Lambda^{\tau}(\tau-) \\
& -i \int_{N \tau}^{t} U(t-s) F(s) \mathrm{d} s-i U(t-N \tau) \sigma_{N \tau}^{\tau} \int_{(N-1) \tau}^{N \tau} U(N \tau-s) F(s) \mathrm{d} s \\
& +\ldots-i U(t-N \tau) \sigma_{N \tau}^{\tau} U(\tau) \ldots \sigma_{\tau}^{\tau} \int_{0}^{\tau} U(\tau-s) F(s) \mathrm{d} s,
\end{aligned}
$$

where $\sigma_{k \tau}^{\tau}=\left(\phi_{k \tau}^{\tau}\right)^{-t}, \phi_{k \tau}^{\tau}$ being the polar factor of $\psi^{\tau}(k \tau-)$. Now, to recover an expression similar to (61), we have to calculate the commutators between the free Schrödinger evolution operator and the multiplication operators by $\sigma_{k \tau}^{\tau}$. The estimates of these commutators are not known for non-smooth $\sigma_{k \tau}^{\tau}$.

Proof. Since $\psi^{\tau}$ is solution of the Schrödinger-Poisson system in the space-time slab $[N \tau,(N+1) \tau) \times \mathbb{R}^{3}$, then we can write

$$
\nabla \psi^{\tau}(t)=U(t-N \tau) \nabla \psi^{\tau}(N \tau+)-i \int_{N \tau}^{t} U(t-s) F(s) \mathrm{d} s,
$$

where $F$ is defined in the statement of the Lemma 25. Now there exists a piecewise smooth function $\theta_{N}$, as specified in the proof of the Lemma 17 , such that

$$
\psi(N \tau+)=e^{i(1-\tau) \theta_{N}} \sqrt{\rho_{n}},
$$

and furthermore all the estimates of the Lemma 17 hold, with $\psi=$ $\psi^{\tau}(N \tau-), \tilde{\psi}=\psi^{\tau}(N \tau+)$ and $\varepsilon=2^{-N} \tau\left\|\psi_{0}\right\|_{H^{1}\left(\mathbb{R}^{3}\right)}$. Therefore, we have

$$
\nabla \psi^{\tau}(N \tau+)=\nabla \psi^{\tau}(N \tau-)-i \frac{\tau}{\hbar} e^{i(1-\tau) \theta_{N}} \Lambda^{\tau}(N \tau-)+r_{N}^{\tau},
$$


where $\left\|r_{N}^{\tau}\right\|_{L^{2}} \leq \tau\left\|\psi_{0}\right\|_{H^{1}}$. By plugging (64) into (63) we deduce

$$
\begin{aligned}
\nabla \psi^{\tau}(t)=U(t-N \tau) \nabla \psi^{\tau}(N \tau-)-i \frac{\tau}{\hbar} U(t-N \tau)\left(e^{i(1-\tau) \theta_{N}} \Lambda^{\tau}(N \tau-)\right) \\
+U(t-N \tau) r_{N}^{\tau}-i \int_{N \tau}^{t} U(t-s) F(s) \mathrm{d} s .
\end{aligned}
$$

Let us iterate this formula, repeating the same procedure for $\nabla \psi^{\tau}(N \tau-)$, then (61) holds.

At this point we can use the formula (61) to obtain Strichartz estimates for $\nabla \psi^{\tau}$, simply by applying the Theorem 9 to each of the terms in (61). After some computations, the following result holds.

Proposition 28 (Strichartz estimates for $\nabla \psi^{\tau}$ ). Let, $0<T<\infty$, let $\psi^{\tau}$ be as in the previous section, then one has

$$
\left\|\nabla \psi^{\tau}\right\|_{L_{t}^{q} L_{x}^{r}\left([0, T] \times \mathbb{R}^{3}\right)} \leq C\left(E_{0}^{\frac{1}{2}},\left\|\rho_{0}\right\|_{L^{1}\left(\mathbb{R}^{3}\right)}, T\right)
$$

for each admissible pair of exponents $(q, r)$.

Remark 29. As we showed in Remark 26, in the general case the nonhomogeneous term $F$ is slightly different. Anyway by the Strichartz estimates the same result of Proposition 28 holds even in the case of modified non-homogeneous term $F$.

Proof. First of all, let us prove the Proposition 28, for a small time $0<T_{1} \leq T$ and let $(q, r)$ be an admissible pair of exponents. We choose $T_{1}>0$ later. Let $N$ be a positive integer such that $T_{1} \leq N \tau$. By applying the Theorem 9 to the formula (61), we get

$$
\begin{aligned}
\left\|\nabla \psi^{\tau}\right\|_{L_{t}^{q} L_{x}^{r}\left(\left[0, T_{1}\right] \times \mathbb{R}^{3}\right)} \leq & \left\|U(t) \nabla \psi_{0}\right\|_{L_{t}^{q} L_{x}^{r}\left(\left[0, T_{1}\right] \times \mathbb{R}^{3}\right)} \\
& +\frac{\tau}{\hbar} \sum_{k=1}^{N}\left\|U(t-k \tau)\left(e^{i(1-\tau) \theta_{k}} \Lambda^{\tau}(k \tau-)\right)\right\|_{L_{t}^{q} L_{x}^{r}\left(\left[0, T_{1}\right] \times \mathbb{R}^{3}\right)} \\
& +\sum_{k=1}^{N}\left\|U(t-k \tau) r_{k}^{\tau}\right\|_{L_{t}^{q} L_{x}^{r}\left(\left[0, T_{1}\right] \times \mathbb{R}^{3}\right)} \\
& +\left\|\int_{0}^{t} U(t-s) F(s) \mathrm{d} s\right\|_{L_{t}^{q} L_{x}^{r}\left(\left[0, T_{1}\right] \times \mathbb{R}^{3}\right)} \\
=: A+B+C+D . &
\end{aligned}
$$

Now we estimate term by term the above expression.

The estimate of $A$ is straightforward, since

$$
\left\|U(t) \nabla \psi_{0}\right\|_{L_{t}^{q} L_{x}^{r}\left(\left[0, T_{1}\right] \times \mathbb{R}^{3}\right)} \lesssim\left\|\nabla \psi_{0}\right\|_{L^{2}\left(\mathbb{R}^{3}\right)} .
$$


The estimate of $B$ follows from

$$
\begin{aligned}
\frac{\tau}{\hbar} \sum_{k=1}^{N}\left\|U(t-k \tau)\left(e^{i(1-\tau) \theta_{k}} \Lambda^{\tau}(k \tau-)\right)\right\|_{L_{t}^{q} L_{x}^{r}\left(\left[0, T_{1}\right] \times \mathbb{R}^{3}\right)} & \\
& \lesssim \tau \sum_{k=1}^{N}\left\|\Lambda^{\tau}(k \tau-)\right\|_{L^{2}\left(\mathbb{R}^{3}\right)} \lesssim T_{1} E_{0}^{\frac{1}{2}}
\end{aligned}
$$

The term $C$ can be estimated in a similar way, namely

$$
\sum_{k=1}^{N}\left\|U(t-k \tau) r_{k}^{\tau}\right\|_{L_{t}^{q} L_{x}^{r}} \lesssim \sum_{k=1}^{N}\left\|r_{k}^{\tau}\right\|_{L^{2}\left(\mathbb{R}^{3}\right)} \lesssim T_{1}\left\|\psi_{0}\right\|_{H^{1}\left(\mathbb{R}^{3}\right)}
$$

The last term is a little bit more tricky to estimate. First of all we decompose $F$ into three terms, $F=F_{1}+F_{2}+F_{3}$, where $F_{1}=$ $\nabla\left(\left|\psi^{\tau}\right|^{p-1} \psi^{\tau}\right), F_{2}=\nabla V^{\tau} \psi^{\tau}$ and $F_{3}=V^{\tau} \nabla \psi^{\tau}$. By the Strichartz estimates (Theorem 9), we have

$$
\begin{aligned}
& \quad\left\|\int_{0}^{t} U(t-s) F(s) \mathrm{d} s\right\|_{L_{t}^{q} L_{x}^{r}\left(\left[0, T_{1}\right] \times \mathbb{R}^{3}\right)} \\
& \lesssim\left\|F_{1}\right\|_{L_{t}^{q_{1}^{\prime}} L_{x}^{r_{1}^{\prime}}\left(\left[0, T_{1}\right] \times \mathbb{R}^{3}\right)}+\left\|F_{2}\right\|_{L_{t}^{q_{2}^{\prime}} L_{x}^{r_{2}^{\prime}}\left(\left[0, T_{1}\right] \times \mathbb{R}^{3}\right)}+\left\|F_{3}\right\|_{L_{t}^{q_{3}^{\prime}} L_{x}^{r_{3}^{\prime}}\left(\left[0, T_{1}\right] \times \mathbb{R}^{3}\right)},
\end{aligned}
$$

where $\left(q_{i}, r_{i}\right)$ are pairs of admissible exponents. Let us start with the first term.

Lemma 30. There exists $\alpha>0$, depending on $p$, such that

$$
\left\|\left|\psi^{\tau}\right|^{p-1} \nabla \psi^{\tau}\right\|_{L_{t}^{\tilde{q}^{\prime}} L_{x}^{\tilde{r}^{\prime}}\left(\left[0, T_{1}\right] \times \mathbb{R}^{3}\right)} \lesssim T_{1}^{\alpha}\left\|\psi^{\tau}\right\|_{\dot{S}^{1}\left(\left[0, T_{1}\right] \times \mathbb{R}^{3}\right)}
$$

Proof. First of all let us apply Hölder inequality on the left-hand side of (69). Then we have

$$
\begin{aligned}
& \left\|\left|\psi^{\tau}\right|^{p-1} \nabla \psi^{\tau}\right\|_{L_{t}^{\tilde{q}^{\prime}} L_{x}^{\tilde{x}^{\prime}}\left(\left[0, T_{1}\right] \times \mathbb{R}^{3}\right)} \\
& \quad \lesssim T_{1}^{\alpha}\left\|\left|\psi^{\tau}\right|^{p-1}\right\|_{L_{t}^{q_{1}} L_{x}^{r_{1}}\left(\left[0, T_{1}\right] \times \mathbb{R}^{3}\right)}\|\nabla \psi\|_{L_{t}^{q_{2}} L_{x}^{r_{2}}\left(\left[0, T_{1}\right] \times \mathbb{R}^{3}\right)} \\
& \quad=T_{1}^{\alpha}\left\|\psi^{\tau}\right\|_{L_{t}^{q_{1}(p-1)} L_{x}^{r_{1}(p-1)}\left(\left[0, T_{1}\right] \times \mathbb{R}^{3}\right)}\left\|\nabla \psi^{\tau}\right\|_{L_{t}^{q_{2}} L_{x}^{r_{2}}\left(\left[0, T_{1}\right] \times \mathbb{R}^{3}\right)}
\end{aligned}
$$

Now we want $\frac{1}{q_{1}(p-1)}=\frac{3}{2}\left(\frac{1}{6}-\frac{1}{r_{1}(p-1)}\right)$ and $\frac{1}{q_{2}}=\frac{3}{2}\left(\frac{1}{2}-\frac{1}{r_{2}}\right)$, in such a way that $\|f\|_{L_{t}^{q_{1}(p-1)} L_{x}^{r_{1}(p-1)}},\|\nabla f\|_{L_{t}^{q_{2}} L_{x}^{r_{2}}} \leq\|f\|_{\dot{S}^{1}}=\|\nabla f\|_{\dot{S}^{0}}$. We already know $\frac{1}{\tilde{q}^{\prime}}=1+\frac{3}{2}\left(\frac{1}{2}-\frac{1}{\tilde{r}^{\prime}}\right)$, then putting together the conditions on 
$(\tilde{q}, \tilde{r}),\left(q_{j}, r_{j}\right)$, it follows

$$
\begin{aligned}
\frac{1}{\tilde{q}^{\prime}}=\frac{1}{\alpha}+\frac{1}{q_{1}}+\frac{1}{q_{2}}=\frac{1}{\alpha}+(p-1) & \frac{3}{2}\left(\frac{1}{6}-\frac{1}{r_{1}(p-1)}\right) \\
+ & \frac{3}{2}\left(\frac{1}{2}-\frac{1}{r_{2}}\right)=1+\frac{3}{2}\left(\frac{1}{2}-\frac{1}{\tilde{r}^{\prime}}\right)
\end{aligned}
$$

and when $1 \leq p<5$,

$$
\alpha=\frac{5-p}{4}>0
$$

This means that we can always choose pairs $\left(\tilde{q}^{\prime}, \tilde{r}^{\prime}\right),\left(q_{1}, r_{1}\right),\left(q_{2}, r_{2}\right)$ satisfying the previous conditions so that the inequality (70) holds, with $\alpha>0$.

For instance, if $1 \leq p \leq 3$, we can choose $\frac{1}{r_{1}}=\frac{p-1}{6}, \frac{1}{r_{2}}=\frac{1}{2}$, therefore $q_{1}=q_{2}=\infty$, hence we have $\frac{1}{\tilde{r}^{\prime}}=\frac{2+p}{6}, \frac{1}{\tilde{q}^{\prime}}=\frac{5-p}{4}$. In the case $3 \leq p<5$, we take $\frac{1}{r_{1}}=\frac{p-1}{6}, \frac{1}{r_{2}}=\frac{1}{6}$ then $q_{1}=\infty, q_{2}=2$, hence we have $\frac{1}{\tilde{r}^{\prime}}=\frac{p}{6}, \frac{1}{\tilde{q}^{\prime}}=\frac{7-p}{4}$.

Now let us consider the second term: $V^{\tau} \nabla \psi^{\tau}$, here we choose $\left(q_{2}^{\prime}, r_{2}^{\prime}\right)=$ $(1,2)$, so by the Hölder and the Hardy-Littlewood-Sobolev (see [49]) inequalities one has

$$
\begin{aligned}
\left\|V^{\tau} \nabla \psi^{\tau}\right\|_{L_{t}^{1} L_{x}^{2}\left(\left[0, T_{1}\right] \times \mathbb{R}^{3}\right)} \leq T_{1}^{\frac{1}{2}}\left\|V^{\tau}\right\|_{L_{t}^{\infty} L_{x}^{2}}\left\|\nabla \psi^{\tau}\right\|_{L_{t}^{2} L_{x}^{6}} & \\
& \lesssim T_{1}^{\frac{1}{2}}\left\|\psi^{\tau}\right\|_{L_{t}^{\infty} L_{x}^{2}}^{2}\left\|\nabla \psi^{\tau}\right\|_{L_{t}^{2} L_{x}^{6} .}
\end{aligned}
$$

For the third term, we choose $\left(q_{3}^{\prime}, r_{3}^{\prime}\right)=\left(\frac{2}{2-3 \varepsilon}, \frac{2}{1+2 \varepsilon}\right)$ and again by using the Hardy-Littlewood-Sobolev and the Hölder inequalities, we have

$$
\begin{aligned}
& \left\|\nabla V^{\tau} \psi^{\tau}\right\|_{L_{t}^{\frac{2}{2-3 \varepsilon}}} \frac{2}{L_{x}^{1+2 \varepsilon}}\left(\left[0, T_{1}\right] \times \mathbb{R}^{3}\right) \\
& \lesssim T_{1}^{\frac{1}{2}}\left\|\nabla V^{\tau}\right\|_{L_{t}^{\frac{2}{1-3 \varepsilon}} L_{x}^{\frac{1}{\varepsilon}}}\left\|\psi^{\tau}\right\|_{L_{t}^{\infty} L_{x}^{2}} \\
& \lesssim T_{1}^{\frac{1}{2}}\left\|\nabla\left|\psi^{\tau}\right|^{2}\right\|_{L_{t}^{1-3 \varepsilon}} \frac{3}{L_{x}^{2+3 \varepsilon}}\left\|\tilde{\psi}^{\tau}\right\|_{L_{t}^{\infty} L_{x}^{2}} \lesssim T_{1}^{\frac{1}{2}}\left\|\psi^{\tau}\right\|_{L_{t}^{\infty}}^{2} L_{x}^{2}\left\|\nabla \psi^{\tau}\right\|_{L_{t}^{1-3 \varepsilon}} \frac{2}{L_{x}^{1+6 \varepsilon}}
\end{aligned}
$$

Now, we summarize the previous estimates by using (61) in the following way

$$
\begin{gathered}
\text { (71) }\left\|\nabla \psi^{\tau}\right\|_{\dot{S}^{0}\left(\left[0, T_{1}\right] \times \mathbb{R}^{3}\right)} \lesssim\left\|\nabla \psi_{0}\right\|_{L^{2}\left(\mathbb{R}^{3}\right)}+T_{1} E_{0}^{\frac{1}{2}}+T_{1}^{\alpha}\left\|\nabla \psi^{\tau}\right\|_{\dot{S}^{0}\left(\left[0, T_{1}\right] \times \mathbb{R}^{3}\right)}^{p} \\
+T_{1}^{\frac{1}{2}}\left\|\psi_{0}\right\|_{L^{2}\left(\mathbb{R}^{3}\right)}^{2}\left\|\nabla \psi^{\tau}\right\|_{\dot{S}^{0}\left(\left[0, T_{1}\right] \times \mathbb{R}^{3}\right)} \\
\lesssim(1+T) E_{0}^{\frac{1}{2}}+T_{1}^{\alpha}\left\|\nabla \psi^{\tau}\right\|_{\dot{S}^{0}\left(\left[0, T_{1}\right] \times \mathbb{R}^{3}\right)}^{p}+T_{1}^{\frac{1}{2}}\left\|\psi_{0}\right\|_{L^{2}\left(\mathbb{R}^{3}\right)}^{2}\left\|\nabla \psi^{\tau}\right\|_{\dot{S}^{0}\left(\left[0, T_{1}\right] \times \mathbb{R}^{3}\right)} .
\end{gathered}
$$


Lemma 31. There exist $T_{1}\left(E_{0},\left\|\psi_{0}\right\|_{L^{2}\left(\mathbb{R}^{3}\right)}, T\right)>0$ and $C_{1}\left(E_{0},\left\|\psi_{0}\right\|_{L^{2}\left(\mathbb{R}^{3}\right)}, T\right)>$ 0 , independent on $\tau$, such that

$$
\left\|\nabla \psi^{\tau}\right\|_{\dot{S}^{0}\left([0, \tilde{T}] \times \mathbb{R}^{3}\right)} \leq C_{1}\left(E_{0},\left\|\psi_{0}\right\|_{L^{2}\left(\mathbb{R}^{3}\right)}, T\right)
$$

for all $0<\tilde{T} \leq T_{1}\left(E_{0},\left\|\psi_{0}\right\|_{L^{2}\left(\mathbb{R}^{3}\right)}\right)$.

Let us recall that $E^{\tau}(t)=E_{0}$ and $\left\|\psi^{\tau}(t)\right\|_{L^{2}\left(\mathbb{R}^{3}\right)}=\left\|\psi_{0}\right\|_{L^{2}\left(\mathbb{R}^{3}\right)}$, hence we are in the situation in which we can repeat our argument every time interval of length $T_{1}$, depending always on the same parameters $E_{0},\left\|\psi_{0}\right\|_{L^{2}}$. The consequence of this fact is the following inequality on $[0, T]$

$$
\begin{aligned}
& \left\|\nabla \psi^{\tau}\right\|_{\dot{S}^{0}\left([0, T] \times \mathbb{R}^{3}\right)} \\
& \quad \leq C_{1}\left(E_{0},\left\|\psi_{0}\right\|_{L^{2}}, T\right)\left(\left[\frac{T}{T_{1}}\right]+1\right)=C\left(\left\|\psi_{0}\right\|_{L^{2}}, E_{0}, T\right) .
\end{aligned}
$$

Proof of the Lemma 31. Let us consider the non-trivial case $\left\|\psi_{0}\right\|_{L^{2}}>0$. Assume that $X \in(0, \infty)$ satisfies

$$
X \leq A+\mu X+\lambda X^{p}=\phi(X),
$$

with $p>1, A>0$ and for all $0<\mu<1, \lambda>0$. Let $X_{*}$ be such that $\phi^{\prime}\left(X_{*}\right)=1$, namely $X_{*}=\left(\frac{1-\mu}{p \lambda}\right)^{\frac{1}{p-1}}$, hence one has $\phi\left(X_{*}\right)<X_{*}$ each time the following inequality is satisfied

$$
\left(\frac{1}{p^{\frac{1}{p-1}}}-\frac{1}{p^{\frac{p}{p-1}}}\right) \frac{(1-\mu)^{\frac{p}{p-1}}}{\lambda^{\frac{1}{p-1}}}>A .
$$

Therefore the convexity of $\phi$ implies that, if the condition (75) holds, there exist two roots $X_{ \pm}, X_{+}(\mu, \lambda, A)>X_{*}>X_{-}(\mu, \lambda, A)$, to the equation $\phi(X)=X$. It then follows either $0 \leq X \leq X_{-}$, or $X \geq X_{+}$. In our case $\mu=T_{1}^{1 / 2}\left\|\psi_{0}\right\|_{L^{2}}^{2}, \lambda=T_{1}^{\alpha}, A=(1+T) E_{0}^{1 / 2}$, hence we assume

$$
\begin{gathered}
\mu=T_{1}^{1 / 2}\left\|\psi_{0}\right\|_{L^{2}}^{2}<\frac{1}{2} \\
\lambda=T_{1}^{\alpha}=T_{1}^{\frac{5-p}{4}}<\left[\frac{p^{-\frac{1}{p-1}}-p^{-\frac{p}{p-1}}}{2^{\frac{p}{p-1}}(1+T) E_{0}^{1 / 2}}\right]^{p-1} .
\end{gathered}
$$


Therefore we choose

$$
T_{1}:=\min \left[\left(2\left\|\psi_{0}\right\|_{L^{2}}\right)^{-2},\left[\frac{p^{-\frac{1}{p-1}}-p^{-\frac{p}{p-1}}}{2^{\frac{p}{p-1}}(1+T) E_{0}^{1 / 2}}\right]^{\frac{4(p-1)}{5-p}}\right]
$$

Clearly we cannot have

$$
x_{*}=\left[\frac{1-T_{1}\left\|\psi_{0}\right\|_{L^{2}}}{p T_{1}^{\alpha}}\right]^{\frac{1}{p-1}} \leq x_{+} \leq\left\|\nabla \psi^{\tau}\right\|_{\dot{S}^{0}\left(\left[0, T_{1}\right] \times \mathbb{R}^{3}\right)},
$$

since we get a contradiction as $T_{1} \rightarrow 0$, hence

$$
\left\|\nabla \psi^{\tau}\right\|_{\dot{S}^{0}\left(\left[0, T_{1}\right] \times \mathbb{R}^{3}\right)} \leq X_{-}
$$

Corollary 32. Let $0<T<\infty$ and let $\sqrt{\rho^{\tau}}, \Lambda^{\tau}$ be as in previous section, then

$$
\left\|\nabla \sqrt{\rho^{\tau}}\right\|_{L_{t}^{q} L_{x}^{r}\left([0, T] \times \mathbb{R}^{3}\right)}+\left\|\Lambda^{\tau}\right\|_{L_{t}^{q} L_{x}^{r}\left([0, T] \times \mathbb{R}^{3}\right)} \leq C\left(E_{0}^{\frac{1}{2}},\left\|\rho_{0}\right\|_{L^{1}\left(\mathbb{R}^{3}\right)}, T\right),
$$

for each admissible pair of exponents $(q, r)$.

Unfortunately this is not enough to achieve the convergence of the quadratic terms. We need some additional compactness estimates on the sequence $\left\{\nabla \psi^{\tau}\right\}$ in order to apply Theorem 13. In particular we need some tightness and regularity properties on the sequence $\left\{\nabla \psi^{\tau}\right\}$, therefore we apply some results concerning local smoothing due to Vega [52] and Constantin, Saut [9].

Proposition 33 (Local smoothing for $\nabla \psi^{\tau}$ ). Let $0<T<\infty$ and let $\psi^{\tau}$ be defined as in the previous section. Then one has

$$
\left\|\nabla \psi^{\tau}\right\|_{L^{2}\left([0, T] ; H_{l o c}^{1 / 2}\left(\mathbb{R}^{3}\right)\right)} \leq C\left(E_{0}, T,\left\|\rho_{0}\right\|_{L^{1}}\right) .
$$

Proof. Using the Strichartz estimates obtained above, we can apply the Theorems 11, 12 about local smoothing. Indeed, by using again the 
formula (61) it follows

$$
\begin{aligned}
\left\|\nabla \psi^{\tau}\right\|_{L^{2}\left([0, T] ; H_{l o c}^{1 / 2}\left(\mathbb{R}^{3}\right)\right)} \lesssim & \left\|\nabla \psi_{0}\right\|_{L^{2}\left(\mathbb{R}^{3}\right)} \\
& +\frac{\tau}{\hbar} \sum_{k=1}^{N}\left\|\Lambda^{\tau}(k \tau-)\right\|_{L^{2}\left(\mathbb{R}^{3}\right)} \\
& +\tau \sum_{k=1}^{N}\left\|\nabla \psi_{n_{k}}\right\|_{L^{2}\left(\mathbb{R}^{3}\right)} \\
& +\sum_{k=1}^{N}\left\|\nabla \psi_{n_{k}}-\nabla \psi^{\tau}(k \tau-)+\frac{\tau}{\hbar}\left(\Lambda_{n_{k}}-\Lambda^{\tau}(k \tau-)\right)\right\|_{L^{2}\left(\mathbb{R}^{3}\right)} \\
& +\|F\|_{L^{1}\left([0, T] ; L^{2}\left(\mathbb{R}^{3}\right)\right)} .
\end{aligned}
$$

The first three terms are clearly estimated by a constant $C\left(E_{0}, T\right)$ depending only on the initial energy and on time. The fourth term is $O(\tau)$. The last term will be estimated using the previous Strichartz estimates. As before, we split $F$ into three parts $F=F_{1}+F_{2}+F_{3}$, then one has

$$
\begin{aligned}
& \left\|\left|\psi^{\tau}\right|^{p-1} \nabla \psi^{\tau}\right\|_{L_{t}^{1} L_{x}^{2}\left([0, T] \times \mathbb{R}^{3}\right)} \\
& \leq T^{\frac{4}{5-p}}\left\|\left|\psi^{\tau}\right|^{p-1}\right\|_{L_{t}^{\frac{4}{p-1}} L_{x}^{\infty}\left([0, T] \times \mathbb{R}^{3}\right)}\left\|\nabla \psi^{\tau}\right\|_{L_{t}^{\infty} L_{x}^{2}\left([0, T] \times \mathbb{R}^{3}\right)} \\
& \quad \leq T^{\frac{4}{5-p}}\left\|\psi^{\tau}\right\|_{L_{t}^{4} L_{x}^{\infty}\left([0, T] \times \mathbb{R}^{3}\right)}^{(p-1)}\left\|\nabla \psi^{\tau}\right\|_{L_{t}^{\infty} L_{x}^{2}\left([0, T] \times \mathbb{R}^{3}\right)},
\end{aligned}
$$

while

$$
\left\|\nabla V^{\tau} \psi^{\tau}\right\|_{L_{t}^{1} L_{x}^{2}\left([0, T] \times \mathbb{R}^{3}\right)} \leq T^{\frac{1}{2}}\left\|\nabla V^{\tau}\right\|_{L_{t}^{\frac{2}{1-2 \varepsilon}} L_{x}^{\frac{1}{\varepsilon}}\left([0, T] \times \mathbb{R}^{3}\right)}\left\|\psi^{\tau}\right\|_{L_{t}^{\frac{2}{3 \varepsilon}}} L_{x}^{\frac{2}{1-2 \varepsilon}}\left([0, T] \times \mathbb{R}^{3}\right)
$$

and now the remaining calculations are similar to those already done for the Strichartz estimates. Regarding the term $V^{\tau} \nabla \psi^{\tau}$ we already estimated its $L_{t}^{1} L_{x}^{2}$ norm.

Since $H_{l o c}^{1 / 2}$ is compactly embedded in $L_{l o c}^{2}$, we can apply the Theorem 13 due to Rakotoson, Temam [44].

Proposition 34. The sequence $\left\{\nabla \psi^{\tau}\right\}$ is strongly convergent in $L^{2}\left([0, T] ; L_{l o c}^{2}\left(\mathbb{R}^{3}\right)\right)$, namely

$$
\nabla \psi:=s-\lim _{k \rightarrow \infty} \nabla \psi^{\tau_{k}} \quad \text { in } L^{2}\left([0, T] ; L_{l o c}^{2}\left(\mathbb{R}^{3}\right)\right) .
$$

In particular, one has $\nabla \sqrt{\rho^{\tau}} \rightarrow \nabla \sqrt{\rho}$ and $\Lambda^{\tau} \rightarrow \Lambda$ in $L^{2}\left([0, T] ; L_{l o c}^{2}\left(\mathbb{R}^{3}\right)\right)$. 
Proof. The previous Proposition 33 implies that the sequence $\left\{\nabla \psi^{\tau}\right\}_{\tau>0}$, is uniformly bounded in $L^{2}\left([0, T] ; H_{l o c}^{1 / 2}\left(\mathbb{R}^{3}\right)\right)$ and then $\nabla \psi^{\tau} \rightarrow \nabla \psi$ in that space. Now $H_{l o c}^{1 / 2}$ is compactly embedded in $L_{l o c}^{2}$, since $\nabla \psi^{\tau}(t) \rightarrow$ $\nabla \psi(t)$ for almost every $t \geq 0$ and

$$
\lim _{|E| \rightarrow 0, E \subset[0, T]} \sup _{\tau>0} \int_{E}\left\|\nabla \psi^{\tau}(t)\right\|_{L_{l o c}^{2}}^{2} \mathrm{~d} t=0,
$$

since $\nabla \psi^{\tau} \in L^{\infty}\left([0, T] ; L^{2}\left(\mathbb{R}^{3}\right)\right)$. Hence we can apply the Theorem 13 by Rakotoson and Temam [44] and we get (80).

Proposition 35. $(\rho, J)$ is a weak solution to the Cauchy problem (1), (2).

Proof. It follows directly by combining the Theorem 23 in the section 5 and the Proposition 34. As for the collisionless QHD system we should note that the generalized irrotationality condition holds by the definition of the current density and Corollary 16.

Acknowledgement. The authors wish to thank prof. Luigi Ambrosio for some useful comments.

\section{REFERENCES}

[1] Ambrosio L., Transport Equations and Cauchy Problem for BV Vector Fields, Inventiones Mathematicae 158, 227-260 (2004).

[2] Ancona M. Iafrate G., Quantum correction to the equation of state of an electron gas in a semiconductor, Phys. Rev. B 39, pp. 9536-9540 (1989).

[3] Aubin J.-P., Un Théorème de compacité, C. R. Acad. Sci. 256, 5042-5044 (1963).

[4] Ben Abdallah N. Méhats F. Schmeiser C. Weishäupl R. M., The nonlinear Schrödinger equation with a strongly anisotropic harmonic potential, SIAM J. Math. Anal. 37, no. 1, 189-199 (2005).

[5] Brenier Y., Polar Factorization and Monotone Rearrangement of VectorValued Function, Comm. Pure Appl. Math. 44, 375-417 (1991).

[6] Brezis H., How to recognize constant functions. Connections with Sobolev spaces, Russ. Math. Surveys, 57, n.4, 693 (2002).

[7] Carlen E., Conservative Diffusions, Comm. Math. Phys. 94, 293-315 (1984).

[8] Cazenave T., Semilinear Schrödinger Equations, Courant Lecture Notes in Mathematics, 10. New York University, Courant Institute of Mathematical Sciences, AMS, 2003. 
[9] Constantin P. Saut J.C., Local Smoothing Properties of Dispersive Equations, J. Amer. Math. Soc. 1 (1988), 413-439.

[10] Cheng N.-H. Shatah J. Uhlenbeck K., Schrödinger Maps, Comm. Pure Appl. Math. 53, 590-602 (2000).

[11] Colliander J. Keel M. Staffilani G. Takaoka H. Tao T., Global well-posedness and scattering for the energy-critical nonlinear Schrödinger equation in $\mathbb{R}^{3}$, to appear Annals.

[12] Dalfovo F. Giorgini S. Pitaevskii L. Stringari S, Theory of Bose-Einstein condensation in trapped gases, Rev. Mod. Phys., 71, 463-512 (1999).

[13] Degond P. Gallego S. Méhats F., Isothermal quantum hydrodynamics: derivation, asymptotic analysis, and simulation, Multiscale Model. Simul. 6, 1, 246272 (2007).

[14] Degond P. Gallego S. Méhats F., On quantum hydrodynamic and quantum energy transport models, Commun. Math. Sci. 5, 4, 1-22 (2007).

[15] Degond P. Ringhofer C., Quantum moment hydrodynamics and the entropy principle, J. Stat. Phys. 112, 587-628 (2003).

[16] Di Perna R.J. Lions P.-L., Ordinary differential equations, transport theory and Sobolev spaces, Invent. Math. 98, 511-547 (1989).

[17] Exner P. Seba P. Sadreev A.F. Streda P. Feher P., Strength of Topologically Induced Magnetic Moments in a Quantum Device, Phys. Rev. Lett., 80, 17101713 (1998).

[18] Federer H. Ziemer W.P., The Lebesgue set of a function whose distribution Derivatives are $p$-th power summable, Indiana Univ. Math. J., 22, 2, 139-158 (1972).

[19] Feynman R.P., Superfluidity and Superconductivity, Rev. Mod. Phys., 29, n.2, 205 (1957).

[20] Gardner C., The Quantum Hydrodynamic Model for Semiconductor Devices SIAM J. Appl. Math. 54, 409-427 (1994).

[21] Gasser I. Markowich P., Quantum hydrodynamics, Wigner transforms and the classical limit, Asymptot. Anal. 14, no. 2, 97-116 (1997).

[22] Gianazza U. Savaré G. Toscani G., The Wasserstein gradient flow of the Fisher information and the Quantum Drift-Diffusion equation, preprint IMATI-CNR (2006).

[23] Ginibre J. Velo G., The global Cauchy problem for the nonlinear Schrödinger equations revisited, Ann. Inst. H. Poincaré Anal. Non Linéaire 2, 309-327 (1987).

[24] Guerra F. Morato L., Quantization of Dynamical Systems and Stochastic Control Theory, Phys. Rev. D 27, 1771-1786 (1983).

[25] Hayashi N. Nakamitsu K. Tsutsumi M., On Solutions of the Initial Value Problem for the Nonlinear Schrödinger Equations, Journ. Funct. Anal. 71, 218-245 (1987).

[26] Jüngel A. Li H.-L. Matsumura A., The relaxation-time limit in the quantum hydrodynamic equations for semiconductors, J. Diff. Eq. 225, 440-464 (2006).

[27] Jüngel A. Mariani M.C. Rial D., Local Existence of Solutions to the Transient Quantum Hydrodynamic Equations, Math. Models Methods Appl. Sci. 12, no. 4, 485-495 (2002). 
[28] Jüngel A. Matthes D., A derivation of the isothermal quantum hydrodynamic equations using entropy minimization, Z. Angew. Math. Mech. 85, 806-814 (2005).

[29] Jüngel A. Matthes D. Milisic J. P., Derivation of new quantum hydrodynamic equations using entropy minimization, SIAM J. Appl. Math. 67, no. 1, 46-68, (2006).

[30] Hutchinson D.A.W. Zaremba E. Griffin A., Finite Temperature Excitations of a Trapped Bose Gas, Phys. Rev. Lett. 78, 1842 (1997).

[31] Kadanoff L.P. Baym G., Quantum Statistical Mechanics, Benjamin, N.Y., 1962

[32] Khalatnikov I.M., An introduction to the Theory of Superfluidity, Benjamin N.Y., 1965.

[33] Keel M. Tao T., Endpoint Strichartz Estimates, Amer. J. Math. 120 (1998), 955-980.

[34] Kirkpatrick T.R. Dorfman J.R., Transport theory for a weakly interacting condensed Bose gas, Phys. Rev. A 28, 2576 (1983).

[35] Landau L.D., Theory of the Superfluidity of Helium II, Phys. Rev. 60, 356 (1941).

[36] Li H. Lin C.-K., Semiclassical limit and well-posedness of nonlinear Schrödinger-Poisson systems, EJDE 93, 1-17 (2003).

[37] Li H. L. Marcati P., Existence and asymptotic behavior of multi-dimensional quanntum hydrodynamic model for semiconductors, Comm. Math. Phys. 245, 2, 215-247 (2004).

[38] Lifshitz E.M. Pitaevskii L., Physical Kinetics, Pergamon, Oxford (1981).

[39] Lions J.-L., Equations différentialles opérationnells et problèmes aux limites, Springer, Berlin (1961).

[40] Madelung E., Quantentheorie in hydrodynamischer form, Z. Physik, 40, 322 (1927).

[41] Markowich P., Ringhofer C.Quantum hydrodynamics for semiconductors in the high-field case, Appl. Math. Lett. 7, n.5, 37-41 (1994).

[42] Nelson E., Quantum Fluctuations, Princeton University Press, 1984.

[43] Pauli W., in Handbuch der Physik, edited by H. Geiger and K. Scheel (Springer, Berlin, 1933), vol. 24, pt. 1, p. 98.

[44] Rakotoson J.M. Temam R., An Optimal Compactness Theorem and Application to Elliptic-Parabolic Systems, Appl. Math. Letters 14 (2001), 303-306.

[45] Seba P. Kuhl U. Barth M. Stöckmann H-J., Experimental verification of topologically induced vortices inside a billiard, J. Phys. A: Math. Gen., 32, 82258230 (1999).

[46] Shatah J. Struwe M., Geometric wave equations, Courant Lecture Notes in Math., 2 (1998).

[47] Simon J., Compact sets in the space $L^{p}(0, T ; B)$, Ann. Mat. Pura Appl.146, 65-96 (1987).

[48] Sjölin P., Regularity of solutions to the Schrödinger equation, Duke Math. J. 55, n.3, 699-715 (1987).

[49] Sogge C., Fourier integrals in classical analysis, Cambridge Univ. Press (1993)

[50] Tao T, Nonlinear Dispersive Equations: Local and Global Analysis, CBMS regional conference series in mathematics (2006). 
[51] Teufel S. Tumulka R., Simple proof for global existence of bohmian trajectories, Comm. Math. Phys. 258, 349-365 (2005).

[52] Vega L., Schödinger equations: pointwise convergence to the initial data, Proc. AMS 102, n.4, 874-878 (1988).

[53] Weigert S., How to determine a quantum state by measurements: the Pauli problem for a particle with arbitrary potential, Phys. Rev. A 53, 4, 2078-2083 (1996).

[54] Wigner E., On the quantum correction for the thermodynamic equilibrium, Phys. Rev. 40, 749 (1932).

Paolo Antonelli - Dipartimento di Matematica Pura ed Applicata, Università degli Studi dell'Aquila, Via Vetoio, 67010 Coppito (AQ), ITALY

E-mail address: paolo.antonelli@univaq.it

Pierangelo Marcati - Dipartimento di Matematica Pura ed AppliCata, Università degli Studi dell'Aquila, Via Vetoio, 67010 Coppito (AQ), ITALY

E-mail address: marcati@univaq.it 